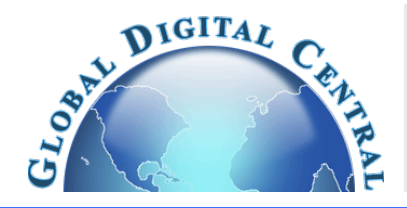

\title{
NATURAL CONVECTON IN SINUSOIDAL-CORRUGTED ENCLOSURE UTITIING SILVER/WATER NANOLUID WITH DIFFERENT SHAPES OF CONCENTRIC INNER CYLINDERS
}

\author{
Emad D. Aboud ${ }^{1}$, Qusay Rasheed Al-Amir², Hameed K. Hamzah², Ammar Abdulkadhim³, Mustafa M. Gabir ${ }^{3}$, \\ Salwan Obaid Waheed Khafaji ${ }^{2}$, Farooq H. Ali ${ }^{*}, 2$ \\ ${ }^{1}$ College of Engineering, Al-Musayab-Autombile Engineering Department, University of Babylon, Babylon, Hilla, Iraq \\ ${ }^{2}$ College of Engineering-Mechanical Engineering Department - University of Babylon-Babylon City-Hilla- Iraq. \\ ${ }^{3}$ Air Conditioning and Refrigeration Techniques Engineering Department - Al-Mustaqbal University College-Babylon City- Iraq.
}

\begin{abstract}
The natural convection of nanofluid flow, which occurs between a sinusoidal-corrugated enclosure and a concentric inner cylinder has been numerically investigated. The two horizontal walls of this enclosure are considered adiabatic and two vertical corrugated walls are held at a constant value of the cold temperature while the inner concentric cylinder is heated isothermally. Different cylinder geometries (i.e, circular, square, rhombus, and triangular) located inside the enclosure are examined to find the best shape for optimum heat transfer. The physical and geometrical parameters influencing heat transfer are Rayleigh number $\left(\mathrm{Ra}=10^{3}-10^{6}\right)$, undulation numbers $(\mathrm{N}=0,1$ and 2$)$, aspect ratios $(\mathrm{AR}=5,2.5$ and 1.67$)$ and two values for the volume fraction ( $\varphi=0$ and 0.05 ). The numerical simulation was carried out using Comsol Multiphysics Software (5.3a). Galerkin approach along with the finite element method are used to solve equations of Navier-Stokes and energy with associated boundary conditions. In this study, validations of results between some available literatures and the present study found to be in an excellent agreement. Results indicate that the heat transfer and nanofluid flow characteristics in the sinusoidal-corrugated enclosure is significantly influenced by aspect ratio, undulation number, and Rayleigh number for all cylinder shapes. Thus, with the decrease in the aspect ratio, the intensity of streamlines becomes smaller, whereas, with rice in the Rayleigh number and undulation number, intensity increase is observed. Moreover, as the undulation number increases, the average value of the Nusselt number, including the hot surface of cylinders increases. At high Rayleigh numbers, the undulation number effect on the average value of the Nusselt number is more pronounced. Besides, the research showed that the circular cylinder shape inside the enclosure has the best heat transfer characteristics and flow than the others.
\end{abstract}

Keywords: Natural convection, Inner cylinder shapes, Undulation number, Aspect ratio, Streamline and isotherm contours, Flow and heat transfer characteristics.

\section{INTRODUCTION}

For several years owing to a wide range of practical applications like heat exchangers, electronic devices cooling, crude oil storage tanks, solar collectors and the nuclear reactor, the topic of the implanted solid body inside enclosures motivated the researchers among the world to deeply examine it (Joudi et al. (2004); Ostrach (1988); Baytos and Pop (1999); Bin et al. (2001), Chamkha et al. (2011)). It was obtained based upon the previous publications, that the solid inserted body within the enclosure improves the heat transfer thermal rate. From the other hand it will decrease the flow, therefore lowering heat transfer inside the enclosures. Also, the effects of three shapes of inner cylinders (circular, square, and triangular) on heat transfer by Hussein et al.(2019). House et al. (1990) conducted computationally the buoyancy thermal driven flow in a square cavity containing conductive square body under transient conditions. It was achieved that the convection mode would be more dominant than conduction at a higher Rayleigh number. Besides that, they compared with and without the inner body and they conclude that inner body affect the heat transfer characteristics. The vertical shift for the location of an internal cylinder of the circular shape located within an enclosure taken the square shape illustrated numerically by Humaira et al. (2002). Hussain and Al-Amir (2011) demonstrated the influence of inner body size located within a square enclosure on the rate of heat transfer. Shu et al., (2001) employed the DQ technique to illustrate the impact of the eccentric annulus between the inner circular cylinder positioned within the square enclosure. Hussain S.H. and Hussein A.K., (2010) examined the impact of the circular inner body movement vertically within a square enclosure. Immersed boundary element method had beeu utilized by Lee et al. (2010) and Kang et al. (2013) to study the natural phenomenon for the square enclosure within circular body. They changed the inner cylinder position horizontally and diagonally. Roslan et al., (2014) analyzed the buoyancy fluid flow between the inner solid polygon placed within the square enclosure using COMSOL numerically. Choi et al. (2014) demonstrated numerically the inner circular position located

\footnotetext{
${ }^{*}$ Corresponding Author. Email: eng.farooq.hassan@uobabylon.edu.iq
} 
inside a rhombus enclosure under unsteady-state conditions. The natural convection occurred between the inner circular cylinder placed within a parallelogrammical enclosure containing nanofluid was investigated by Majdi et al. (2019) . Effects on natural convection flow inside a heated triangular body situated within an air-filled circular cylindrical cavity with different physical parameters such as inclination angle, radius ratio as well as Rayleigh number examined numerically by Xu.et al. (2009). They obtained that the angle of triangular body inclination hsd no effect. The effect of the presence of a hot inner cylinder sited inside a cold square cavity on heat transfer and flow fields for different Rayleigh number values was numerically demonstrated Lee et al.(2010).

Many liquids have poor thermal conductivity such as oils and water, which is a significant limit for heat transfer rate increasing. For this purpose, the motivation to use a small diameter of solid particles in the base fluid is present. The advanced fluid is therefore referred to as nanofluid ( Kherbeet et al. (2014)). In the last two decades, numerous works have analyzed experimentally and numerically that deal with this field for various types of enclosures such as square, rectangular, circular, triangular trapezoidal, annulus, and C-shaped. (Sheremet (2015); Khanafer (2003); Jou and Tzeng (2006); Khoshvaght (2004); Mohammed et al. (2016); Murshed and de Castro (2016); Tiwari and Das (2007); Celli (2013); Kumar et al. (2010); Aminossadati and Ghasemi (2009); Colangelo et al. (2017); Khoshvaght-Aliabadi et al. (2017)). An investigation using experimental rig presented by Ridha et al. (2013) used different types of nanoparticles inside a square cavity whose vertical walls were partially heated. It is noted that when the Rayleigh number $(\mathrm{Ra})$ was raised, the use of nanoparticle volume fraction improved the heat transfer rate. Ghasemi and Aminossadati (2010) examined the periodic natural heat convection within a nanofluids-filled cavity. The oscillating heat flux source was placed on the left wall of the cavity, whereas the right wall was cold. The other walls were assumed to be adiabatic. Their findings showed that the use of $\mathrm{Cu}$ nanoparticles improves the thermophysical properties, especially at the lower value of the Ra number. A numerical study by Ghasemi and Aminossadati (2010) worked on natural convection for Ethylene Glycol-Copper nanofluid inside a right-angle triangular cavity, the cavity bottom wall was partially heated from the bottom wall. They demonstrated that at high Rayleigh number values, the optimum value of solid fractions of nanoparticles exists. Recently, Ahmed et al. (2018) have analytically studied the thermally driven heat transfer and flow through a rectangular nanofluids vertical conduit.

Because of its important applications in the cooling of electronic devices, complex enclosure geometries such as wavy shape were also studied. The wavy walls can play an important role in addition to nanotechnology in augmenting the heat transfer rate (Al-Amir et al. (2017); Khoshvaght-Aliabadi et al. (2014); Shenoy et al., (2016). The natural convection in a nanofluid wavy enclosure discussed by Cho and Chen (2012), and Esmaeilpour and Abdollahzadeh (2012). The results indicated that the $\mathrm{Nu}$ increases as the volume fraction of the nanofluid increases. Cho et al. (2016) conducted numerically the entropy generation and natural convection in an inclined wavy enclosure filled with A12O3. It is obtained that the Nusselt number of the wavy surface is higher than that for regular surface and when the nanoparticles volume fraction increases, entropy generation decreases. Shirvan et al. (2017) studied the cosine corrugated enclosure filled with nanofluid. Sheikholeslami et al. (2018) used a finite element control volume approach so as to model $\mathrm{A} 12 \mathrm{O} 3$ nanofluid magnetohydrodynamics between the inner corrugated body located inside a porous medium. Another demonstration for the natural flow presented by Sheikholeslami and Oztop (2017) in a complex wavy nanofluid cavity. Alsabery et al. (2018): Hussain and Abd-Amer (2011) examined numerically the mixed convection between the inner solid rotating cylinder concentrically located within a wavy enclosure filled with a porous medium. The effect of magnetic strength and heat radiation on natural convection of nanofluids within movement vertical plate was investigated numerically by Atuti et al. (2019). The simulation is a practical cooling applications of engineering systems. Rafiq Manna and Patric Oosthuizen (2019) presented an experimental and numerical work deals with natural convection from inclined diagonally of two sided square walls having different thickness. Mohmud and Rawand (2020) performed a numerical result for natural convection of square enclosure containing two hot circular cylinder with different displacement vertically and horizontally and variable opening of inlet and outlet port. The results show that Nusselt number decrease when the distance increase horizontally between cylinders for all opening port arrangement.

From the above cited publications, it can be seen that there is limitations on investigations focusing on the influences of shape and aspect ratio of the inner cylinder, undulation number of enclosure vertical walls on the heat transfer and fluid flow within the enclosure filled with silver/water nanofluid. This paper will be the first paper that expresses the problem in detail. Besides, this study is an attempt to identify the best type of inner shape for optimum heat transfer. Therefore, different cylinder geometries with different areas (i.e., circular, square, rhombus and triangular) are examined and discussed. Also, the fluid flow and heat transfer characteristics have been investigated by calculating the local and average $\mathrm{Nu}$ along with the inner cylinder placed inside the center of the enclosure.

\section{PHYSICAL MODEL}

The geometric problem configuration is illustrated in Fig. 1. At the cold temperature of the constant value $(\mathrm{Tc})$, the enclosure vertical walls are maintained, while the other walls are adiabatic. At a constant hot temperature (Th), the concentric inner cylinder located inside the enclosure is held. It is assumed that the vertical wavy walls follow a sinecurve profile.

$Y=\frac{\lambda \sin (N y)}{L}+Y_{o}$

The space between the enclosure walls and the inner cylinder is filled with silver/water nanofluid. The thermal equilibrium between nanoparticles and the fluid is assumed and the diameter of nanoparticles is uniform. Moreover, the thermal conductivity and heat capacity of the nanofluid are constant.

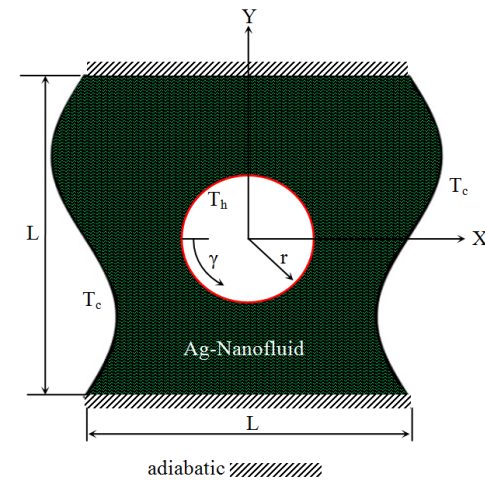

Fig. 1 Computation model of the present model 
The fluid flow in the enclosure is considered to be 2D, steady, laminar, and incompressible. Viscous dissipation is neglected and buoyancy force is treated by Boussinesq approximation. Besides, the radiation effect is neglected between the enclosure inner cylinder and walls. Here, the aspect ratio $(A R=L / 2 R)$ represents the ratio of the length $(\mathrm{L})$ of the wavy enclosure to the diameter $(2 \mathrm{R})$ of the inner shape. In this study, three different aspect ratios $(\mathrm{AR}=5,2.5$, and 1.67) are considered.

The governing differential equations of fluid mechanics and HT are inserted below in the non-dimensional formula (LEE et al. (2010)):

$$
\begin{gathered}
X=\frac{x}{L}, Y=\frac{y}{L}, U=\frac{u L}{\alpha_{f l}}, V=\frac{v L}{\alpha_{f l}}, P=\frac{p L^{2}}{\rho_{f l} \alpha_{f l}}, T^{*}=\frac{T-T_{c}}{T_{h}-T_{c}}, \\
\alpha_{n f l}=\frac{k_{n f l}}{\rho_{n f l}\left(c_{p}\right)_{n f l}}, P r=\frac{v_{f l}}{\alpha_{f l}}, \quad R a=\frac{g \beta_{f l}\left(T_{h}-T_{c}\right) L^{3}}{\alpha_{f l} v_{f l}}
\end{gathered}
$$

With the Boussinesq approximation in y-direction, the continuity, momentum and energy equations in dimensionaless forms are as follows (CHO et al. (2016)):

$$
\begin{aligned}
& \frac{\partial U}{\partial X}+\frac{\partial V}{\partial Y}=0 \\
& \mathrm{U} \frac{\partial U}{\partial X}+V \frac{\partial U}{\partial Y}=-\frac{\partial P}{\partial X}+\frac{\mu_{n f l}}{\rho_{n f l} \alpha_{f l}}\left(\frac{\partial^{2} U}{\partial X^{2}}+\frac{\partial^{2} U}{\partial Y^{2}}\right) \\
& \mathrm{U} \frac{\partial V}{\partial X}+V \frac{\partial V}{\partial Y}=-\frac{\partial P}{\partial Y}+\frac{\mu_{n f l}}{\rho_{n f l} \alpha_{f l}}\left(\frac{\partial^{2} V}{\partial X^{2}}+\frac{\partial^{2} V}{\partial Y^{2}}\right)+\frac{(\rho \beta)_{n f l}}{\rho_{n f l} \beta_{f l}} \operatorname{RaPr} \theta \\
& \mathrm{U} \frac{\partial T^{*}}{\partial X}+V \frac{\partial T^{*}}{\partial Y}=\frac{\alpha_{n f l}}{\alpha_{f l}}\left(\frac{\partial^{2} T^{*}}{\partial X^{2}}+\frac{\partial^{2} T^{*}}{\partial Y^{2}}\right)
\end{aligned}
$$

The thermophysical properties of the nanofluid, such as the heat capacity, density, and coefficient of thermal expansion, can be obtained. These formulas have been used in recent paper (SHEIKHOLESLAMI et al. (2018)) for the determination of the nanofluid thermophysical properties in simulation analysis of natural convection.

$\rho_{n f l}=(1-\varphi) \rho_{f l}+\varphi \rho_{s p}$

$\left(\rho c_{p}\right)_{n f l}=(1-\varphi)\left(\rho c_{p}\right)_{f l}+\varphi\left(\rho c_{p}\right)_{s p}$

$(\rho \beta)_{n f l}=(1-\varphi)(\rho \beta)_{f l}+\varphi(\rho \beta)_{s p}$

According to the Maxwell formula, the nanofluid thermal conductivity for nanoparticles can be given by, The Maxwell formulation is a best estimation and easiness for spherical nanoparticle shape in low concentration.

$k_{n f l}=\frac{\left(k_{s p}+2 k_{s p}\right)-2 \varphi\left(k_{f l}-k_{s p}\right)}{\left(k_{f l}+2 k_{s p}\right)+\varphi\left(k_{f l}+k_{s p}\right)} k_{f l}$

The Brinkman model is employed for estimation of the effective dynamic viscosity of the nanofluid

$$
\mu_{n f l}=\frac{\mu_{f l}}{(1-\varphi)^{2.5}}
$$

The subscripts $(s p)$ and $(f l)$ in the preceding description refer to nanoparticles dispelled and pure fluid, respectively. As stated in SHIRVAN et al., (2017) the thermo-physical properties of water and the studied nanoparticles in this study are given in Table 1.

Table 1. Thermo-physical of the selected working fluid.

\begin{tabular}{|c|c|c|c|c|c|}
\hline Properties & $\begin{array}{c}\mathrm{C}_{\mathrm{p}} \\
(\mathrm{J} / \mathrm{kg} \mathrm{k})\end{array}$ & $\begin{array}{c}\rho \\
\left(\mathrm{kg} / \mathrm{m}^{3}\right)\end{array}$ & $\begin{array}{c}\mathrm{K} \\
(\mathrm{W} / \mathrm{m} . \mathrm{k})\end{array}$ & $\begin{array}{c}\mathrm{B} \\
(1 / \mathrm{k})\end{array}$ & $\begin{array}{c}\mu \\
(\mathrm{kg} / \mathrm{m} . \mathrm{s})\end{array}$ \\
\hline $\begin{array}{c}\text { Pure } \\
\text { water }\end{array}$ & 4179 & 997.1 & 0.613 & $21 \times 10^{-5}$ & 0.000372 \\
\hline Ag & 235 & 429 & 10500 & $1.89 \times 10^{-5}$ & - \\
\hline
\end{tabular}

The local $\mathrm{Nu}$ on around the inner cylinders can be given:

$$
N u_{l}=-\frac{k_{n f l}}{k_{f l}} \frac{\partial T^{*}}{\partial n}
$$

The average Nusselt number $\left(\mathrm{Nu}_{\mathrm{ave}}\right)$ is obtained by integration local Nusselt number around the centered hot inner cylinders.

$$
N u_{\text {ave }}=\frac{1}{l} \int_{0}^{l} N u_{l}(\theta) d \theta
$$

The BCs are described as follows.

Vertical adiabatic

Walls: $U=V=0, \frac{\partial T^{*}}{\partial Y}=0$.

Horizontal cold walls:

$U=V=0, T^{*}=0$

Inner hot cylinder of different shapes (Circle, Rhombic, Square, Triangle):

$$
U=V=0, T^{*}=1
$$

The penalty finite element scheme are used to resolve equations of momentum and energy $(4,5,6)$ by replacing the pressure term $(\mathrm{P})$ by the equation of the penalty term $(\gamma)$ as follows:

$P=-\gamma\left(\frac{\partial U}{\partial X}+\frac{\partial V}{\partial Y}\right)$

Insert the above equation in the momentum equations (4\&5) then we get:

$$
\begin{gathered}
\mathrm{U} \frac{\partial U}{\partial X}+V \frac{\partial U}{\partial Y}=\gamma\left(\frac{\partial^{2} U}{\partial X^{2}}+\frac{\partial^{2} V}{\partial X \partial Y}\right)+\frac{\mu_{n f l}}{\rho_{n f l} \alpha_{f l}}\left(\frac{\partial^{2} U}{\partial X^{2}}+\frac{\partial^{2} U}{\partial Y^{2}}\right) \\
\mathrm{U} \frac{\partial V}{\partial X}+V \frac{\partial V}{\partial Y}=\gamma\left(\frac{\partial^{2} U}{\partial X \partial Y}+\frac{\partial^{2} V}{\partial Y^{2}}\right)+\frac{\mu_{n f l}}{\rho_{n f l} \alpha_{f l}}\left(\frac{\partial^{2} V}{\partial X^{2}}+\frac{\partial^{2} V}{\partial Y^{2}}\right) \\
+\frac{(\rho \beta)_{n f l}}{\rho_{n f l} \beta_{f l}} \operatorname{RaPr} \theta
\end{gathered}
$$

Equation of continuity (2) is consequentially contented for high values of the penalty parameter $(\gamma)$. The value of penalty parameter that produce steady resolved are $10^{7}$.

Extracting the components of dimensionless velocities (U, V) and dimensionless temperature $\left(\mathrm{T}^{*}\right)$ utilizing the original form $\left\{\Gamma_{I}\right\}_{I=1}^{M}$

$U \approx \sum_{I=1}^{M} \mathrm{U}_{I} \Gamma_{I}(X, Y), \quad V \approx \sum_{I=1}^{M} V_{I} \Gamma_{I}(X, Y), \quad T^{*} \approx \sum_{I=1}^{M} T_{I}^{*} \Gamma_{I}(X, Y)$

The Galerkin finite element scheme produces the nonlinear residual terms for Eqn. 6, X-momentum (Eq. 18) Y-momentum (Eq. 19) at every node of the interior domain ( $\vartheta$ (REDDY, 1994).

The fluid flow pattern inside the enclosure is expressed utilizing the stream function $(\Psi)$ expression which is derived from the components of velocities ( $\mathrm{U}$ and $\mathrm{V}$ ). The equations are related between the stream function and components of velocities which are expressed in the following equations.

$$
U=\frac{\partial \Psi}{\partial Y}, V=-\frac{\partial \Psi}{\partial X}
$$

Which produce the following equation

$\frac{\partial^{2} \Psi}{\partial X^{2}}+\frac{\partial^{2} \Psi}{\partial Y^{2}}=\frac{\partial U}{\partial Y}-\frac{\partial V}{\partial X}$

Considering the above equation to describe the signs of stream function leads to that negative sign refers to clockwise direction while positive sign maintains the anti-clockwise direction of recirculation flow. Extracting the equation of stream function with the original form $\left\{\Gamma_{I}\right\}_{I=1}^{M}$ as:

$$
\Psi \approx \sum_{I=1}^{M} \Psi_{I} \Gamma_{I}(X, Y)
$$

The relation of $U \& V$ are taken from the equation (20). 
The local Nusselt number around the cylinders $\left(\mathrm{Nu}_{1}\right)$ are described by the relation:

$$
N u_{l}=\sum_{j=1}^{9} T_{j}^{*} \frac{\partial \Gamma_{j}}{\partial n}
$$

The average $\mathrm{Nu}$ around the cylinders are evaluated by integration the local $\mathrm{Nu}$ around the circumference of the cylinders as explained in equation (13).

\section{NUMERICAL SOLUTION}

The finite element method based upon the Galerkin approach is used to solve the dimensionless governing equations with the boundary conditions numerically. The Galerkin method produces nonlinear residual equations as listed below for equations $(18,19 \& 3)$, for each node of the interior domain:

$$
\begin{aligned}
& R_{j}^{(1)}=\sum_{I=1}^{M} U_{I} \int_{\Omega}\left[\left(\sum_{I=1}^{M} U_{I} \Gamma_{I}\right) \frac{\partial \Gamma_{I}}{\partial X}+\left(\sum_{I=1}^{M} V_{I} \Gamma_{I}\right) \frac{\partial \Gamma_{I}}{\partial Y}\right] \Gamma_{I} d X d \\
& +\gamma\left[\sum_{I=1}^{M} U_{I} \int_{\Omega} \frac{\partial \Gamma_{i}}{\partial X} \frac{\partial \Gamma_{I}}{\partial X} d X d Y\right. \\
& \left.+\sum_{I=1}^{M} V_{I} \int_{\Omega} \frac{\partial \Gamma_{i}}{\partial X} \frac{\partial \Gamma_{I}}{\partial Y} d X d Y\right] \\
& +\frac{\mu_{n f l}}{\rho_{n f l} \alpha_{f l}} \sum_{I=1}^{M} U_{I} \int_{\Omega}\left[\frac{\partial \Gamma_{i}}{\partial X} \frac{\partial \Gamma_{I}}{\partial X}\right. \\
& \left.+\frac{\partial \Gamma_{i}}{\partial Y} \frac{\partial \Gamma_{I}}{\partial Y}\right] d X d Y \\
& R_{j}^{(2)}=\sum_{I=1}^{M} V_{I} \int_{\Omega}\left[\left(\sum_{I=1}^{M} U_{I} \Gamma_{I}\right) \frac{\partial \Gamma_{I}}{\partial X}+\left(\sum_{I=1}^{M} V_{I} \Gamma_{I}\right) \frac{\partial \Gamma_{I}}{\partial Y}\right] \Gamma_{I} d X d Y \\
& +\gamma\left[\sum_{I=1}^{M} U_{I} \int_{\Omega} \frac{\partial \Gamma_{i}}{\partial Y} \frac{\partial \Gamma_{I}}{\partial X} d X d Y\right. \\
& \left.+\sum_{I=1}^{M} V_{I} \int_{\Omega} \frac{\partial \Gamma_{i}}{\partial Y} \frac{\partial \Gamma_{I}}{\partial Y} d X d Y\right] \\
& +\frac{\mu_{n f l}}{\rho_{n f l} \alpha_{b f}} \sum_{I=1}^{M} V_{I} \int_{\Omega}\left[\frac{\partial \Gamma_{i}}{\partial X} \frac{\partial \Gamma_{I}}{\partial X}+\frac{\partial \Gamma_{i}}{\partial Y} \frac{\partial \Gamma_{I}}{\partial Y}\right] d X \\
& -\frac{(\rho \beta)_{n a}}{\rho_{n f l} \beta_{f l}} \operatorname{RaPr} \int_{\Omega}\left(\sum_{I=1}^{M} T_{I}^{*} \Gamma_{I}\right) \Gamma_{i} d X d Y
\end{aligned}
$$

$$
\begin{aligned}
R_{j}^{(3)}=\sum_{I=1}^{M} T_{I}^{*} \int_{\Omega}[ & \left.\left(\sum_{I=1}^{M} U_{I} \Gamma_{I}\right) \frac{\partial \Gamma_{I}}{\partial X}+\left(\sum_{I=1}^{M} V_{I} \Gamma_{I}\right) \frac{\partial \Gamma_{I}}{\partial Y}\right] \Gamma_{I} d X d Y \\
& +\frac{\alpha_{n f l}}{\alpha_{f l}} \sum_{I=1}^{M} T_{I}^{*} \int_{\Omega}\left[\frac{\partial \Gamma_{I}}{\partial X} \frac{\partial \Gamma_{i}}{\partial X}+\frac{\partial \Gamma_{I}}{\partial Y} \frac{\partial \Gamma_{I}}{\partial Y}\right] d X d Y
\end{aligned}
$$

The residual equations integral is evaluated by utilizing the Biquadratic basic function of three points Gaussian quadrature. In Eqs. (23 $\&$ 24) penalty parameter $(\gamma)$ can be seen in the second term and is calculated by using two points. For information about the method of reducing the integration of the penalty, the formulation can find in Reddy (2012). N-R technique is utilized to find the solution to the non-linear residual equations (23 \& 24). For more detail, see reference BASAK (2006).

Figure (2) presents the non-uniform mesh distribution of different internal cylinder configurations. Figure (3) shows the grid independency for different configuration (circle, square, triangle and rhombic) at $\mathrm{Ra}=10^{6}, \mathrm{~N}=1, \varphi=0.05$, and $\mathrm{AR}=2.5$.

Before considering the cavity with the undulated wall, the square case is tested under the same conditions and compared to the data as shown in figures ( $4 \& 5$ ). Figure (4) displays the comparison for streamlines and isothermal lines while figure (5) presents the average $\mathrm{Nu}$ with the $\mathrm{Ra}$ comparison for the. The excellent match can be seen between the results obtained from the code and published a paper by Tanmay Basak and Ali J. Chamkha (2012)].

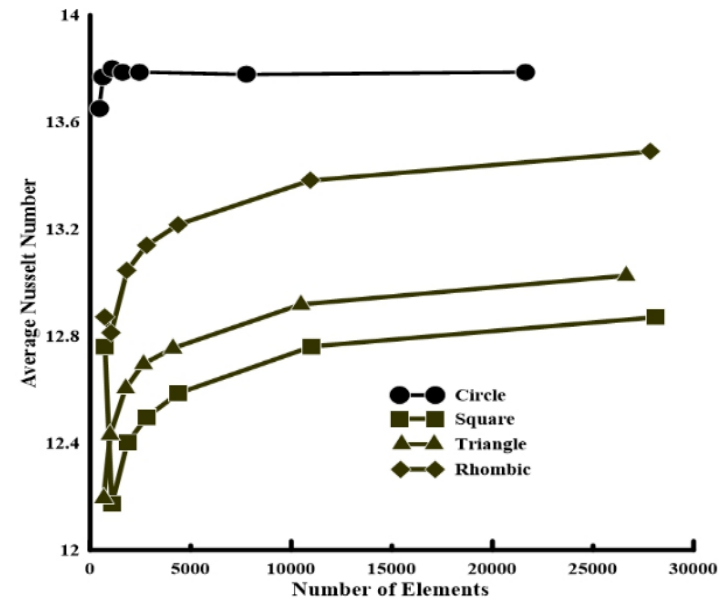

Fig. 3 Mesh validation for different shapes of inner cylinders at $\mathrm{Ra}=10^{6}, \mathrm{~N}=1, \varphi=0.05, \mathrm{AR}=2.5$
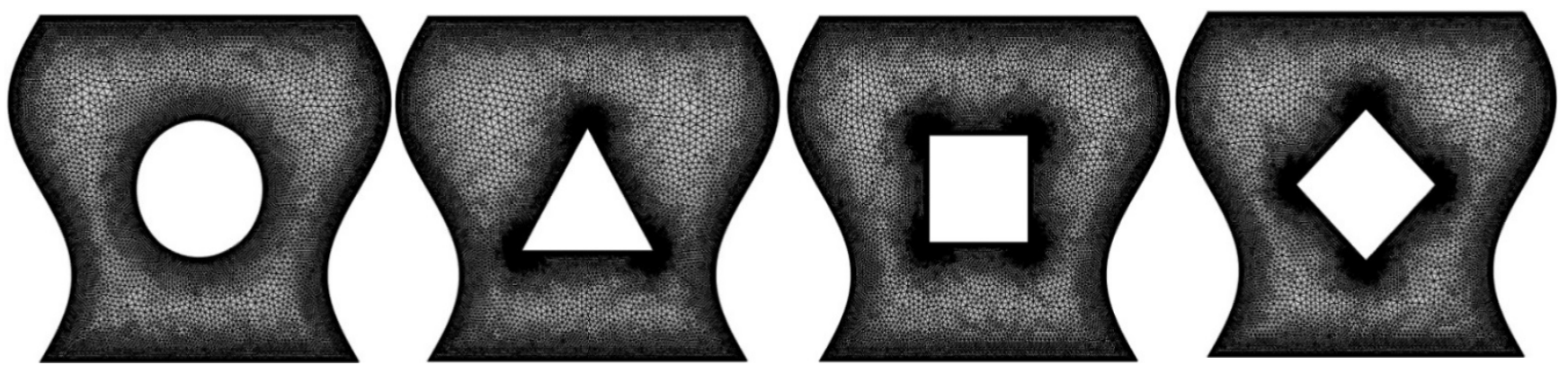

Fig. 2 Mesh generation for different internal cylinder configurations 


\section{Isotherms}
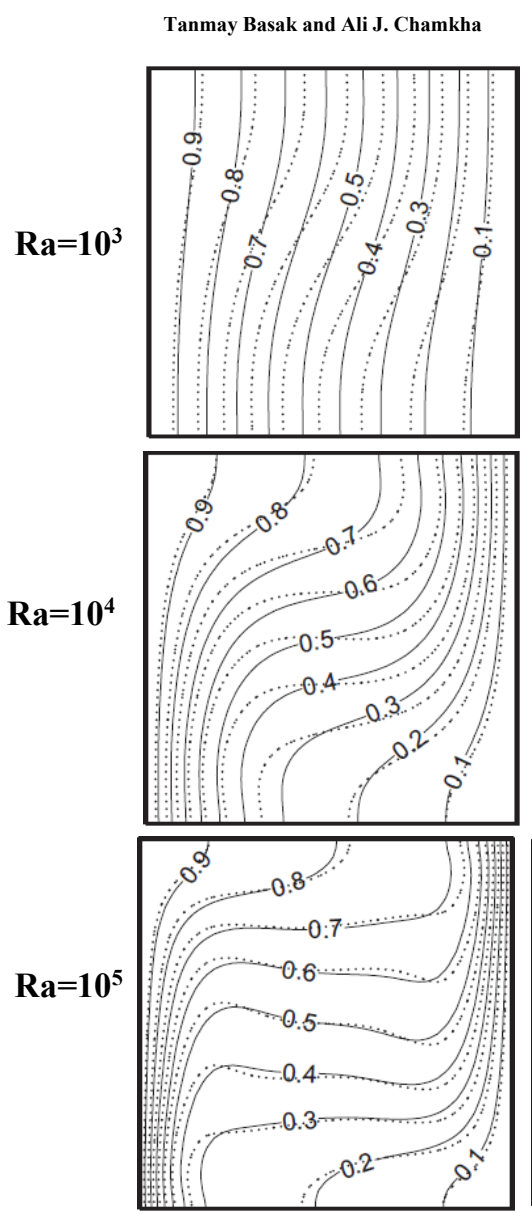
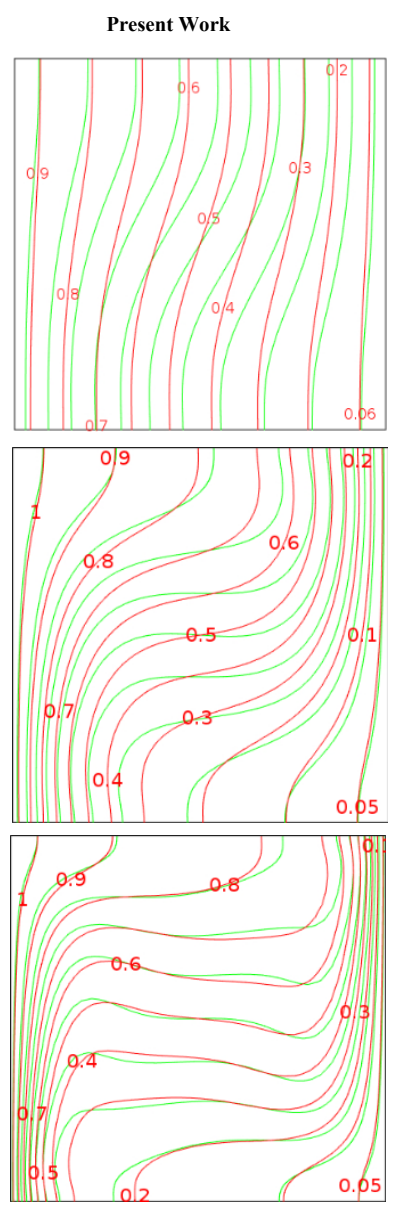

Streamlines
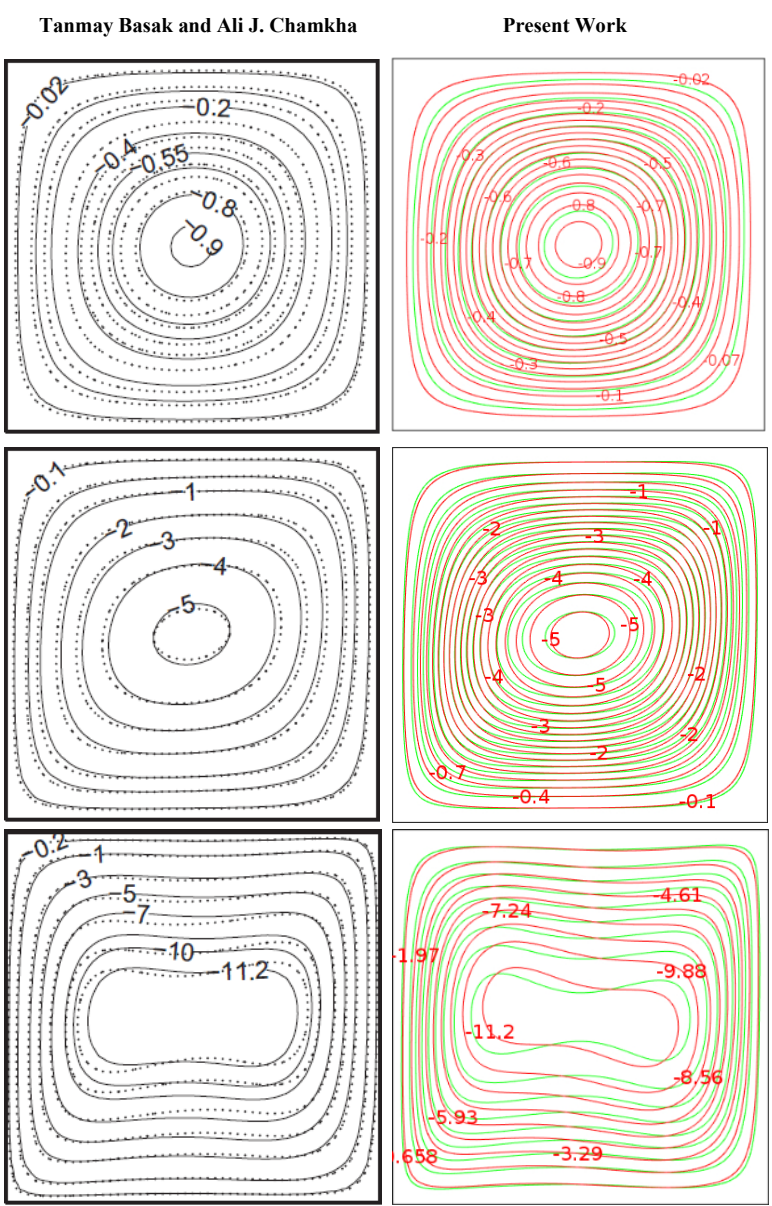

Fig. 4 Comparison of Streamlines and Isotherms between present work and Tanmay Basak and Ali J. Chamkha (2012).
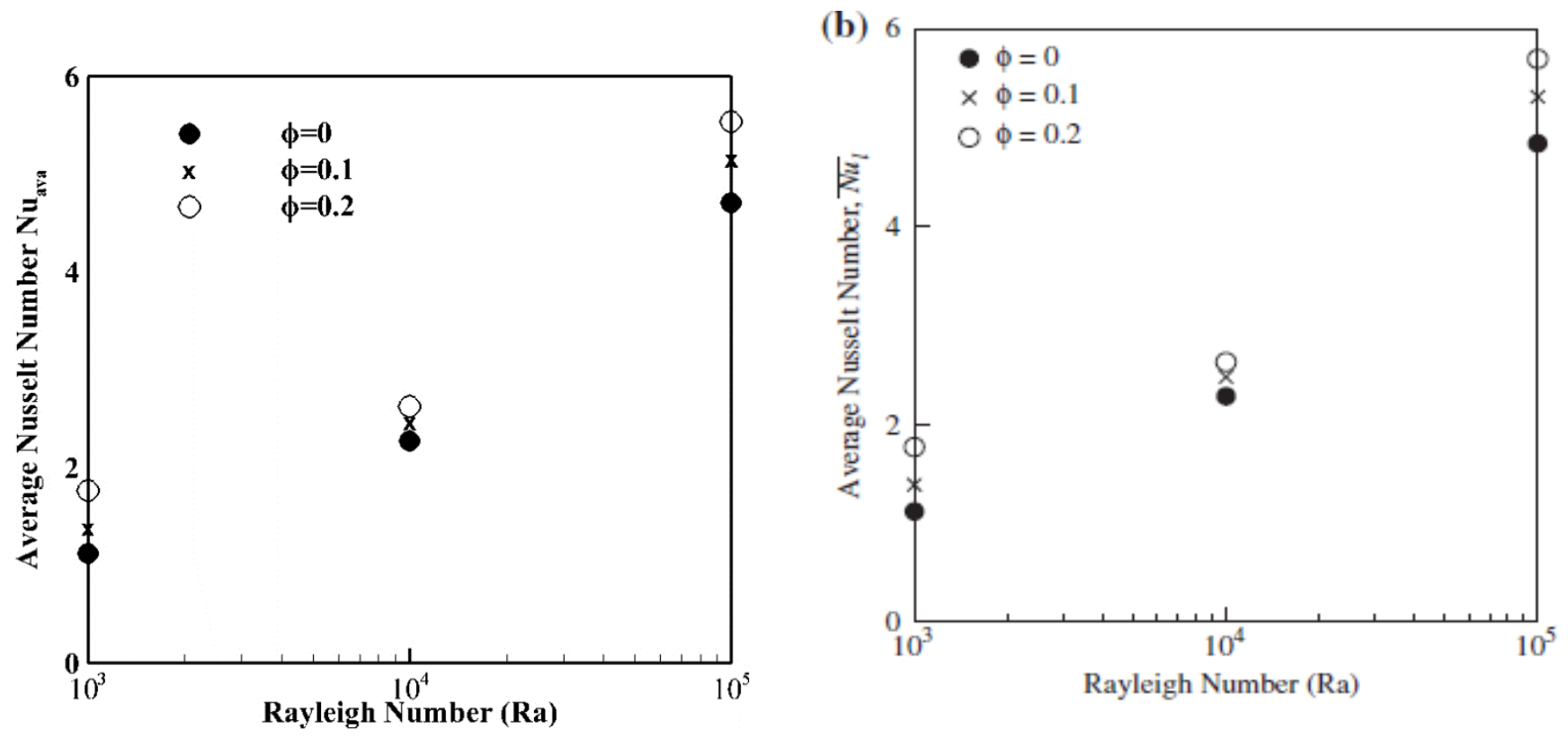

Fig. 5 Comparison of Average Nu between (a) present work and (b) Tanmay Basak and Ali J. Chamkha (2012) 


\section{RESULTS AND DISCUSSIONS}

In this section, the effects of different shapes of inner cylinders on $\mathrm{NC}$ inside a sinusoidal and corrugated enclosure utilizing silver/water nanofluid are investigated for the ranging of the associated dimensionless groups. There are Rayleigh number $\left(\mathrm{Ra}=10^{3}-10^{6}\right)$, undulation numbers $(\mathrm{N}=0,1$ and 2$)$ and aspect ratios $(\mathrm{AR}=5,2.5$ and 1.67). The effects of the inner cylinder shapes namely, a circular, a square, a rhombus and a triangular with different areas are investigated in this study. Owing to the symmetrical BCs along the Y-axis, all the contours of streamlines and isotherms are symmetrical along the vertical centerline of the enclosure. Therefore, the right and left sides of these contours represent plots of isotherms and streamlines, respectively. For various inner cylinder forms, aspect ratios, and $\mathrm{Ra}$, the local and average $\mathrm{Nu}$ are also plotted.

\subsection{Isotherms and streamlines}

In general, all the streamline patterns show that, due to thermal expansion, the heated fluid adjoining to the inner cylinder rises and moves in the upward direction close to the vertical mid-plane of the enclosure until it meets the top cold wall. Then the fluid there becomes progressively cooler and travels horizontally outwards. Consequently, the cooled fluid falls along the cold sides of the walls, then rises again along with the inner cylinder hot surface. Two primary eddies of opposite strength are formed symmetrically in the free space between the inner cylinder and enclosure walls. The shape of the eddies is curved by the presence of the inner cylinder and wavy walls.

"In Figure 6, the effect of the Rayleigh number on the streamline and isothermal contours is shown at $\mathrm{AR}=5, \mathrm{~N}=0$, and $\varphi=0.05$ for different inner cylinder shapes. At $\mathrm{Ra}=10^{3}$, the fluid motion is weak inside the enclosure due to its lower effect on the buoyancy flow.
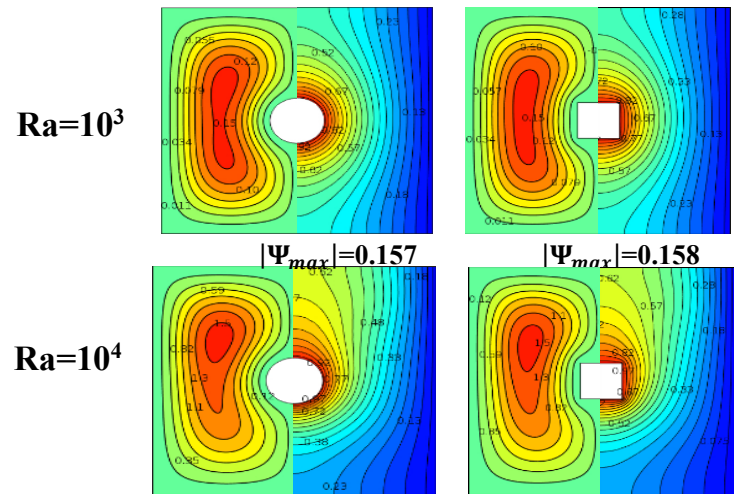

$\left|\Psi_{\text {max }}\right|=\mathbf{0 . 1 5 8}$
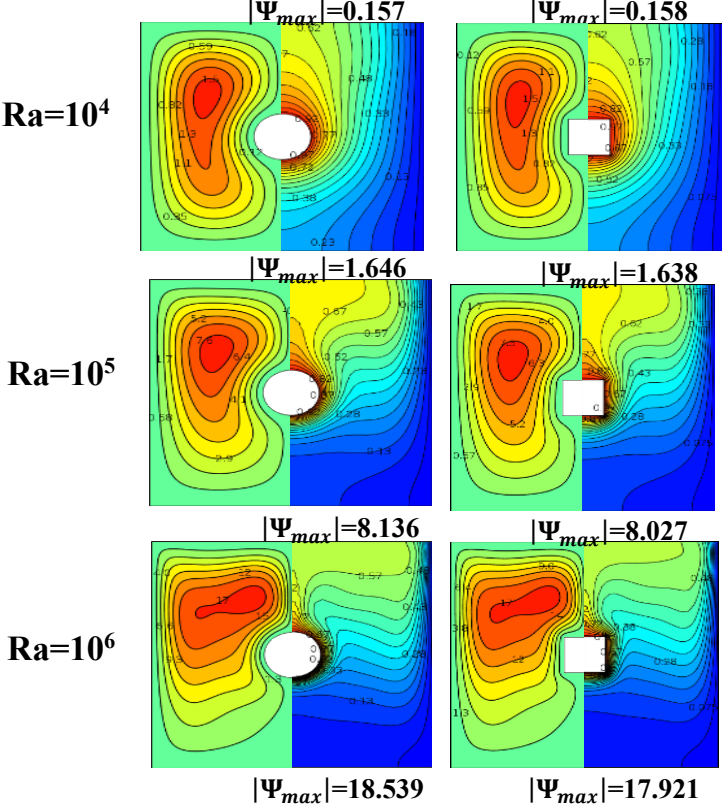

Therefore, the conduction mode is mainly dominated by heat transfer inside the enclosure. The patterns of streamline within the enclosure tend to appear one primary eddy in both sides of the enclosure with different behaviors of inner vortex depending on the cylinder shapes namely, one inner longitudinal vortex for circular and square cylinders, two vortices for rhombus cylinder and one vortex for the triangular cylinder. At $\mathrm{Ra}=$ $10^{4}$, buoyancy forces increase enough to activate significant convection. The patterns of the streamline show that the inner vortices are merged into the primary rotating eddy and move slightly towards the y-axis for all cylinder shapes. The values of the maximum stream function are also increased more with increasing of the Rayleigh number $\left(\Psi_{\max }=0.157\right.$ to 1.646 for a circular shape, $\Psi_{\max }=0.158$ to 1.638 for a square shape, $\Psi_{\max }=0.160$ to 1.687 for a rhombus shape and $\Psi_{\max }=0.186$ to 1.967 for a triangular shape). On the other hand, isotherms at low Ra become dense adjacent to the inner cylinders due to a reduction of convection currents in the enclosure.

The convection currents become more considerable as the Ra increases to $10^{5}$. The heat transfer by convection becomes more important and the warmer fluid goes up through the vertical symmetry line and the thermal boundary layer becomes thinner on the surfaces of the inner cylinder shapes. Consequently, a plume starts to appear on the top of the inner cylinders. In consequence, the dominant flow is in the upper half of the enclosure, and correspondingly the core of the recirculating eddies is located only in the upper half. Therefore, the difference in the values of the maximum stream function becomes more ( $\Psi_{\max }=8.136$ for a circular shape, $\Psi_{\max }=8.027$ for a square shape, $\Psi_{\max }=$ 8.180 for a rhombus shape and $\Psi_{\max }=9.026$ for a triangular shape. At Ra $=10^{6}$, the flow strengthens and the maximum stream function values increase more $\left(\Psi_{\max }=18.539\right.$ for a circular shape, $\Psi_{\max }=17.921$ for a square shape, $\Psi_{\max }=18.940$ for a rhombus shape, and $\Psi_{\max }=20.180$ for a triangular shape). This is because the flow is reinforced at the top surface of the inner cylinder as illustrated in all shapes. On the other hand, a strong plume shows that the flow powerfully impinges on the top of the enclosure, which also improves the transfer of heat

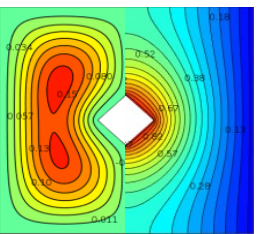

$\left|\Psi_{\max }\right|=0.160$
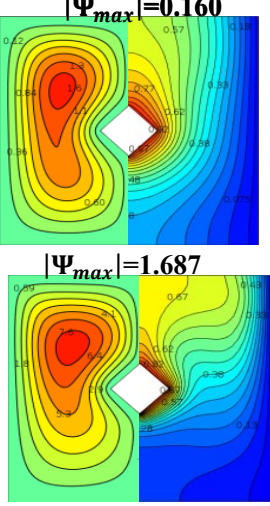

$\left|\Psi_{\max }\right|=\mathbf{8 . 1 8 0}$

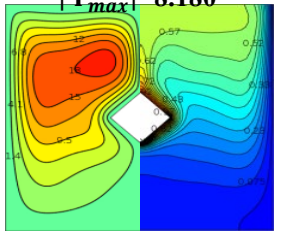

$\left|\Psi_{\max }\right|=18.940$
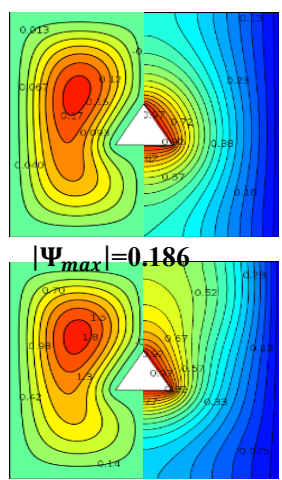

$\left|\Psi_{\max }\right|=1.967$

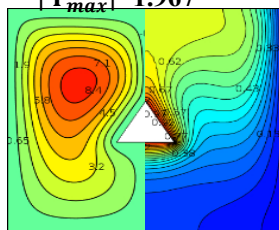

$\left|\Psi_{\max }\right|=9.026$

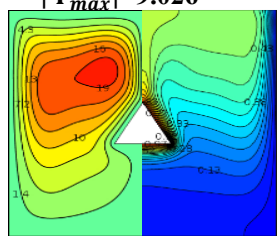

$\left|\Psi_{\max }\right|=20.180$

Fig. 6 Streamlines (left) Isotherms (right) for a different Rayleigh number and geometry of inner cylinders at AR=5, $\mathrm{N}=0, \varphi=0.05$ 
Figure 7 displays the streamline and isotherm contours for various values of the Rayleigh number $\left(\mathrm{Ra}=10^{3}-10^{6}\right)$ at $\mathrm{N}=0$, and $\varphi=0.05$, as the aspect ratio is decreased to $A R=2.5$. In the case of 2.5, buoyancy forces are more reduced as compared with viscous forces for all cylinder shapes. The reason for this is due to a reduced flow rate of fluid in the distance between the cylinder and the enclosure. Consequently, the heat transfer rate by both conduction and convection reduces with a comparison with the previous state when $\mathrm{AR}=5$. For $\mathrm{Ra}=10^{3}$, the maximum streamlines functions reduce with decreasing aspect ratio from 5 to 2.5 for all cylinder's shapes ( $\Psi_{\max }=0.157$ to 0.097 for a circular shape, $\Psi_{\max }=0.158$ to 0.093 for a square shape, $\Psi_{\max }=0.160$ to 0.115 for a rhombus shape and $\Psi_{\max }=0.186$ to 0.164 for a triangular shape). When $\mathrm{Ra}=10^{4}$, the fluid flow rate is still low and not strong enough to augment the convection mode. The isotherms lines are clustered around the hot surface for whole inner cylinder shapes. At $\mathrm{Ra}=10^{5}$, the conduction heat transfer effect decreases due to the increase of flow circulation more than that for the previous state. Therefore, the buoyancy force increases over the viscous force. of enclosure walls due to the convection currents. The buoyancy forces become more considerable as the Ra increases to $10^{6}$. For this reason, the difference in the values of the $\Psi \max$ becomes more $\left(\Psi_{\max }=17.661\right)$ for a circular shape, $\left(\Psi_{\max }=17.616\right)$ for a square shape, $\left(\Psi_{\max }=17.791\right)$ for a rhombus shape and $\left(\Psi_{\max }=19.479\right)$ for a triangular shape. As seen in all cylinder forms, the nuclei of the eddy travel upwards inside the gap between the cylinder and the enclosure due to increased buoyancy. For different Rayleigh number values $\left(\mathrm{Ra}=10^{3}-10^{6}\right)$ at $\mathrm{N}=0$, and $\varphi=0.05$,

Figure 8 indicates the streamline and isothermal contours with a further decrease in the aspect ratio to $\mathrm{AR}=1.67$. In this case, the distance between the walls of the enclosure and the inner cylinder decreases. As a result, temperature gradients increase with declining the size of the primary eddy on both sides of the enclosure. The isotherm and streamline patterns at $\mathrm{Ra}=10^{3}$ are almost symmetric to those at $\mathrm{Ra}=10^{4}$ due to the dominance of the conduction. When Rayleigh number increases $\left(\mathrm{Ra}=10^{5}\right)$, the flow strengthens and the maximum stream function values increase more to those at $\mathrm{Ra}=10^{4}\left(\Psi_{\max }=0.523\right.$ to 4.194$)$ for a circular shape, $(\Psi \max =0.450$ to 3.954$)$ for a square shape, $\left(\Psi_{\max }=0.780\right.$ to 5.432) for a rhombus shape and ( $\Psi_{\max }=1.386$ to 7.996 for a triangular shape).
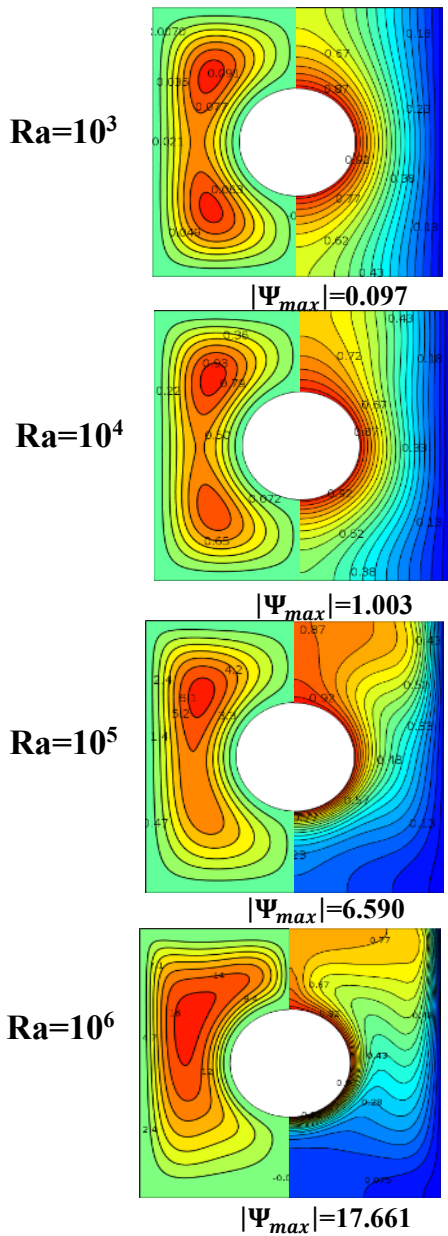

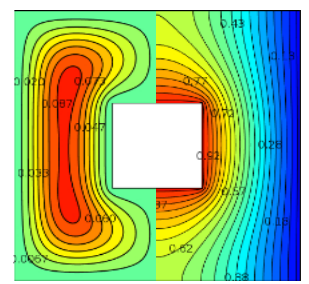

$\Psi_{\text {max }} \mid=0.093$

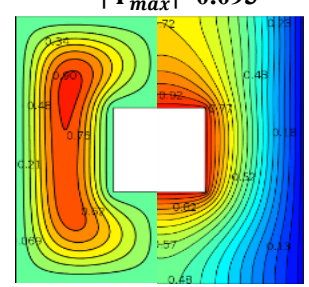

$\left|\Psi_{\text {max }}\right|=0.964$

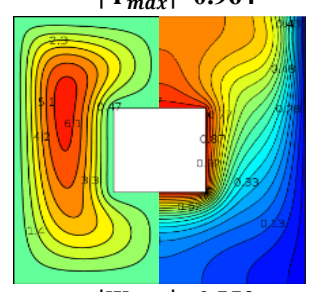

$\left|\Psi_{\text {max }}\right|=6.550$

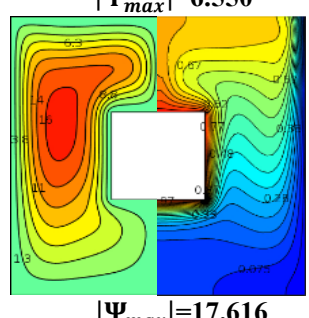

$\left|\Psi_{\max }\right|=17.616$

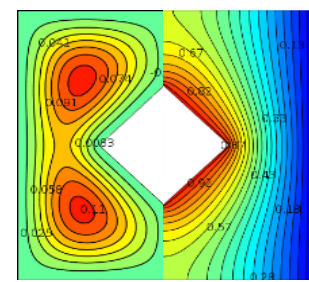

$\left|\Psi_{\text {max }}\right|=0.115$

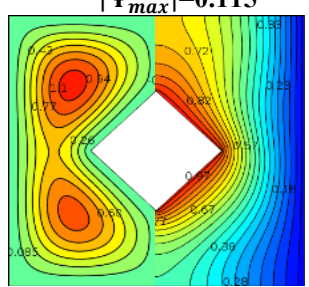

$\left|\Psi_{\max }\right|=1.189$

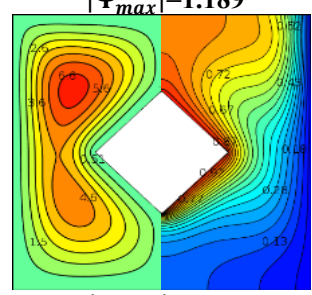

$\left|\Psi_{\max }\right|=7.154$

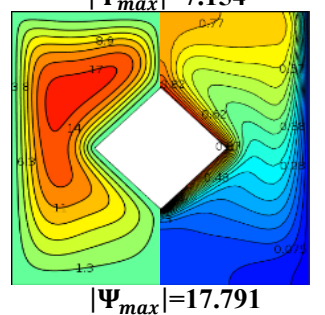

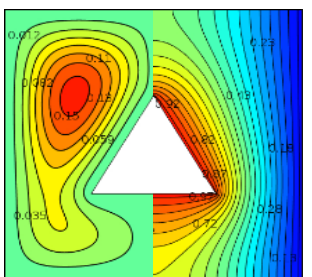

$\left|\Psi_{\text {max }}\right|=0.164$

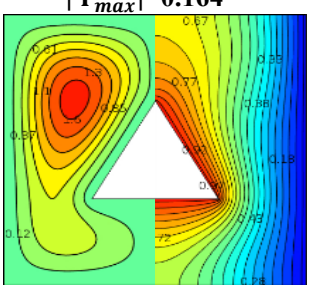

$\left|\Psi_{\max }\right|=1.705$

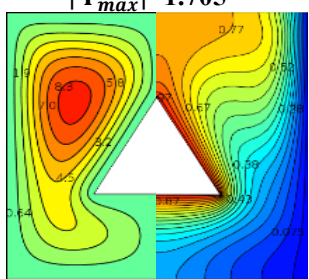

$\left|\Psi_{\text {max }}\right|=8.967$

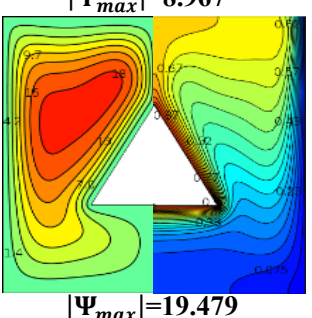

Fig. 7 Streamlines (left) Isotherms (right) for a different Rayleigh number and geometry of inner cylinders at $\mathrm{AR}=2.5, \mathrm{~N}=0, \varphi=0.05$

The values of the maximum stream function are also increased more with increasing of the $\mathrm{Ra}$ from $10^{4}$ to $10^{5}\left(\Psi_{\max }=1.003\right.$ to 6.590 for a circular shape, $\Psi_{\max }=0.964$ to 6.550 for a square shape, $\Psi_{\max }=1.189$ to 7.154 for a rhombus shape and $\Psi_{\max }=1.705$ to 8.967 for a triangular shape). The slope of isotherms contours starts to curve towards the left and right sides
This is because the flow is reinforced at the top inner cylinder surface as illustrated in all shapes. The buoyancy force dominates over the entire domain of flow at $\mathrm{Ra}=10^{6}$. The isothermal curves are highly inclined toward the cooled walls of the enclosure with increasing Ra, which is due to augment energy transmission. 
For various Rayleigh numbers and inner cylinder geometries, the undulation number effect of wavy walls on the streamlines (left) isotherms (right) are presented in Fig. 9 at $\mathrm{AR}=5$ and $\varphi=0.05$. The flow field with wavy walls within the enclosure is comparatively larger than the flow field with smooth walls. Consequently, the fluid flow within the larger field increases, so that the intensity of streamlines is also increased. For example, when $\mathrm{Ra}=10^{6}$ and $\mathrm{AR}=5$, the values of $\Psi_{\max }$ corresponding to $\mathrm{N}=0$ and 1 increase from 18.539 to 19.499 for a circular shape, 17.921 to 18.811 for a square, 18.940 to 19.897 for a rhombus shape, and 18.94 to 21.217 for a triangular shape. We conclude that the values of $\Psi_{\max }$ increase with increasing the number of undulations $(\mathrm{N})$. As shown in fig.9, the patterns of streamline are similar at low $\mathrm{Ra}\left(10^{3}\right.$ and $\left.10^{4}\right)$ due to its lower effects of buoyancy forces inside the enclosure. But with an increase in the Ra, the strength of the stream function increases. The values of $\Psi_{\max }$ corresponding to $\mathrm{Ra}=10^{3}$ and $10^{4}$ are 0.193 and 1.974 for a circular shape, 0.191 and 1.950 for a square shape, 0.196 and 2.003 for a rhombus shape, and 0.218 and 2.237 for a triangular shape. The circulation in the gap between the inner cylinder and the enclosure rises further with the increase of $\mathrm{Ra}\left(10^{5}\right)$, so that the primary eddy rises more than the previous value of $\mathrm{Ra}$ as predicted. The convection currents in the enclosure rise with a further increase of $\mathrm{Ra}$ $\left(10^{6}\right)$, so heat is transferred at a higher rate to the enclosure walls. The resulting flow field undergoes a bifurcation where the primary eddy has two inner vortices. On the other hand, the isotherms collect around the inner cylinder at $\mathrm{Ra}=10^{3}$ due to conduction effects. When $\mathrm{Ra}=10^{4}$, the isotherms shift slightly in the upward direction near the inner shape's left and right sides, reaching the vertical walls of the enclosure.
At the top of inner cylinders, the isotherms become bent because at higher Rayleigh numbers $\left(10^{5}\right.$ to $\left.10^{6}\right)$ the dominance of convection over conduction is observed.

When the aspect ratio decreases to 2.5 , the effects of different inner cylinder geometries and Rayleigh numbers on the streamlines (left) isotherms (right) are illustrated in Fig. 10 at $N=1$ and $\varphi=0.05$. As mentioned before, the results show that decreasing the aspect ratio from 5 to 2.5 leads to reduce the flow field inside the enclosure. As a result, the intensity of the vorticity function is also decreased. The flow field inside the enclosure increases for triangular shape than the rhombus, square and circular shapes, respectively. The reason for this behavior is because the area of the inner triangular shape is smaller than the others. Generally, as Ra increases from $10^{3}$ to $10^{4}$, the streamline patterns are relatively symmetrical for each cylinder shape, although the strength of the vorticity function increases due to the increase in the $\mathrm{Ra}$ ( $\Psi_{\max }=0.141$ to 1.427 for a circular shape, $\Psi_{\max }=0.138$ to 1.403 for square shape, $\Psi_{\max }=0.158$ to 1.588 for rhombus shape and $\Psi_{\max }=0.210$ to 2.086 for a triangular shape). The fluid flow becomes stronger at high $\mathrm{Ra}\left(10^{5}\right.$ and $10^{6}$ ) because of the increased buoyancy force. Owing to the rise in the $\mathrm{Ra}$, the sensitivity of the vorticity function increases. Otherwise, isotherm patterns are changed as $\mathrm{Ra}$ increases from $10^{5}$ to $10^{6}$. In the thermal field, the convection effect becomes more important and the thermal boundary layer at the top of the inner shape becomes thin and at the bottom dense. A plume is also starting to appear on the top of the inner cylinder.
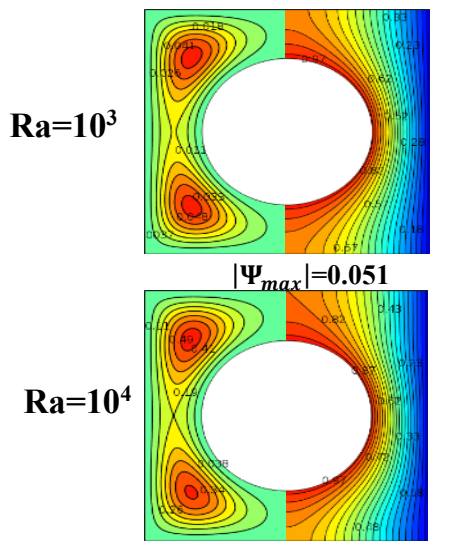

$\left|\Psi_{\max }\right|=0.523$
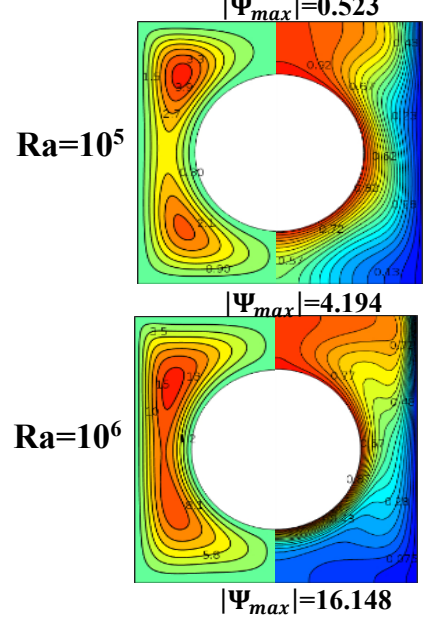

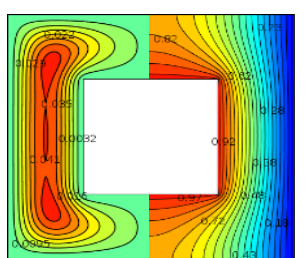

$\left|\Psi_{\max }\right|=\mathbf{0 . 0 4 4}$

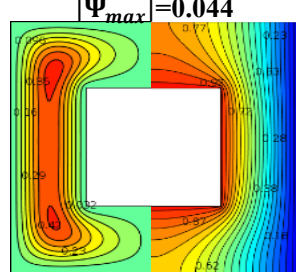

$\left|\Psi_{\max }\right|=\mathbf{0 . 4 5 0}$

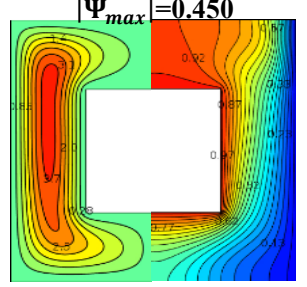

$\left|\Psi_{\max }\right|=3.954$

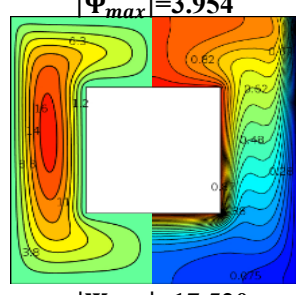

$\left|\Psi_{\text {max }}\right|=17.520$

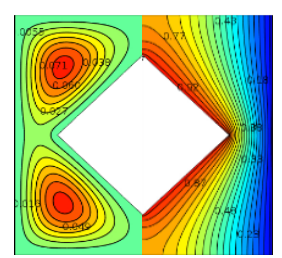

$\left|\Psi_{\max }\right|=0.076$
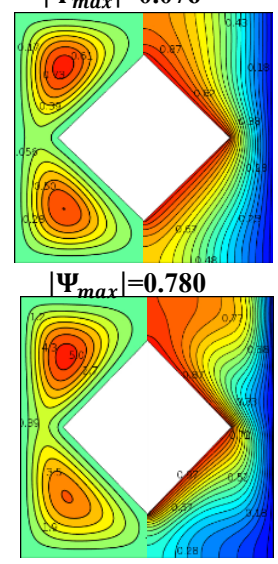

$\left|\Psi_{\max }\right|=\mathbf{5 . 4 3 2}$

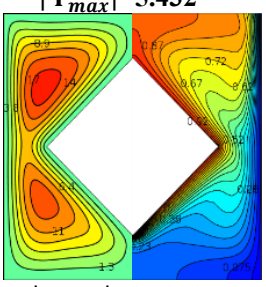

$\left|\Psi_{\max }\right|=17.828$
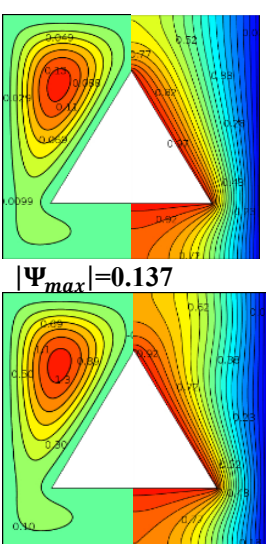

$\left|\Psi_{\max }\right|=1.386$

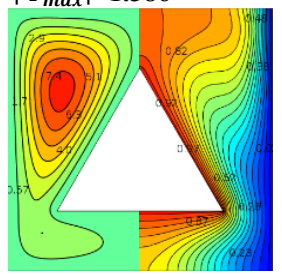

$\left|\Psi_{\max }\right|=7.996$

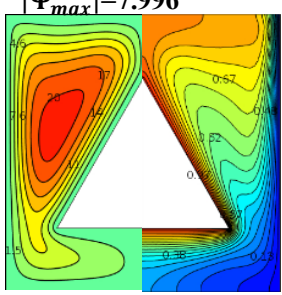

$\left|\Psi_{\text {max }}\right|=21.367$

Fig. 8 Streamlines (left) Isotherms (right) for a different Rayleigh number and geometry of inner cylinders at $\mathrm{AR}=1.67, \mathrm{~N}=0, \varphi=0.05$ 

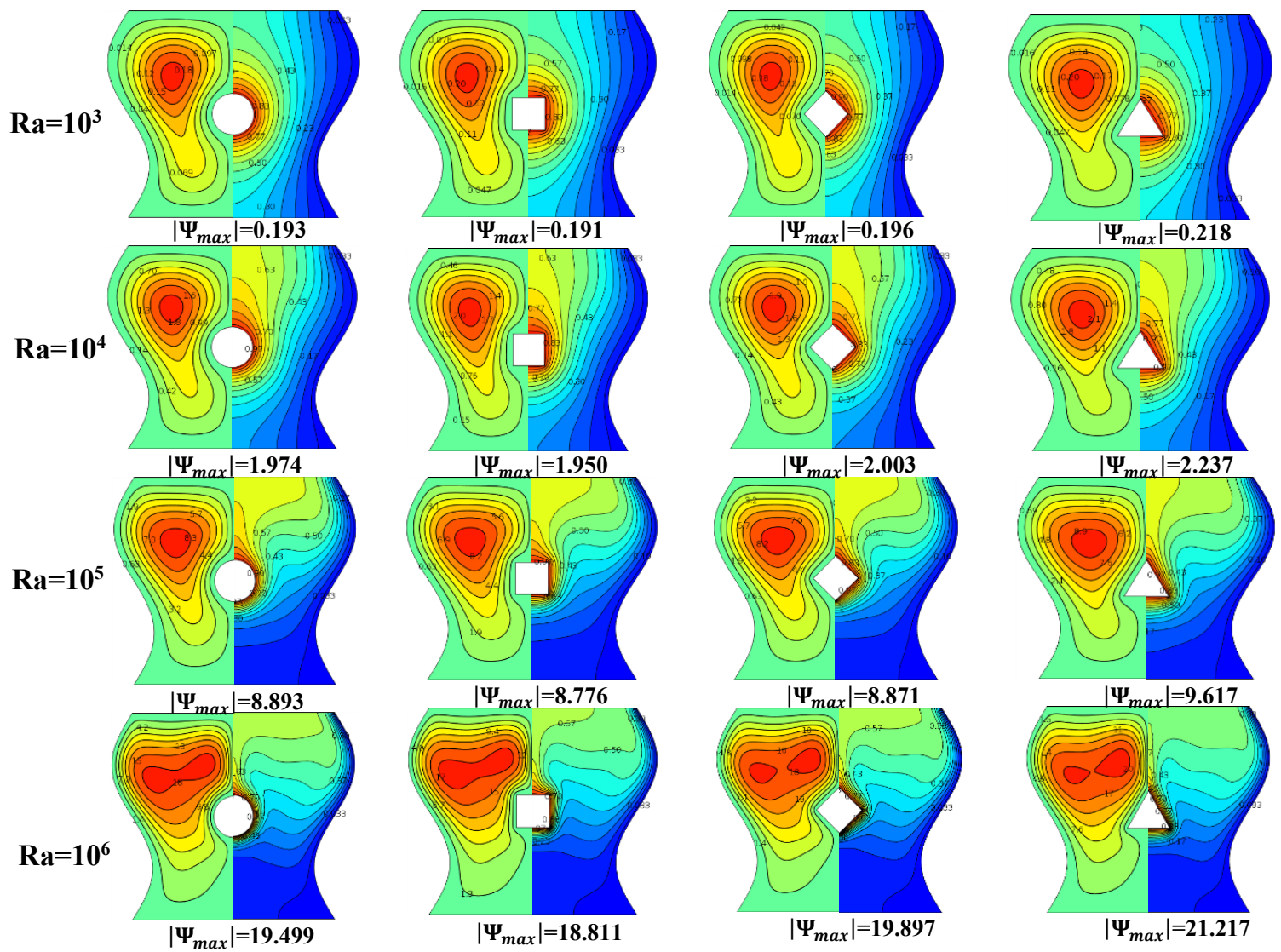

Fig. 9 Streamlines (left) Isotherms (right) for a different Rayleigh number and geometry of inner cylinders at AR=5, $\mathrm{N}=1, \varphi=0.05$
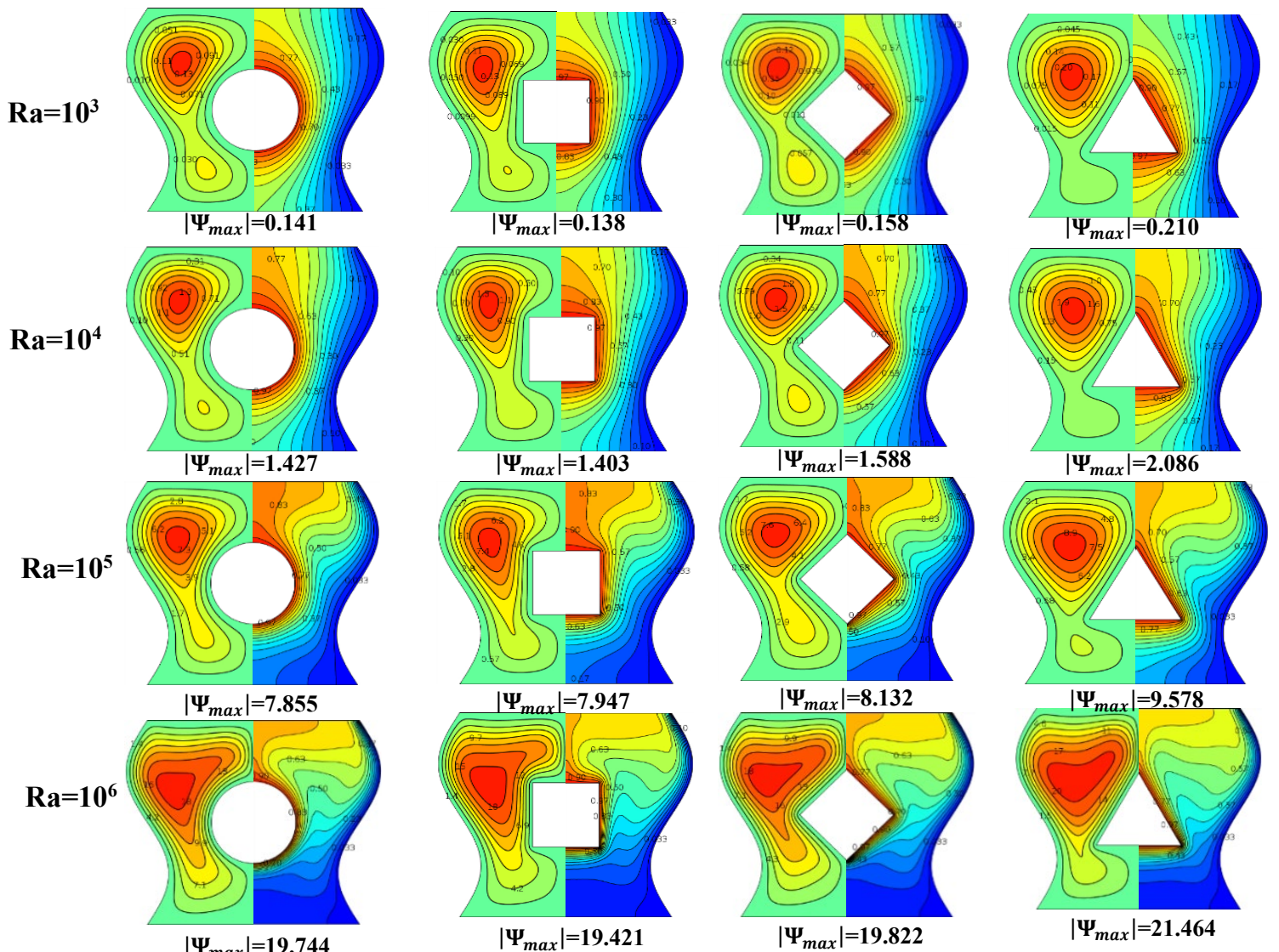

Fig. 10 Streamlines (left) Isotherms (right) for different Rayleigh number and geometry of inner cylinders at $A R=2.5$, $\mathrm{N}=1, \varphi=0.05$ 
The streamline and isotherm patterns have the same behavior with a further decreasing aspect ratio to 1.67 as shown in fig. 11 . The circulation of the flow is also very weak, and the maximum stream function values are small with increasing Rayleigh numbers from $10^{3}$ to $10^{4}$ (i.e., 0.085 to 0.867 for a circular shape, 0.138 to 1.403 for a square shape, 0.114 to 1.151 for a rhombus shape, and 0.137 to1.386 for a triangular shape). The flow displays one primary eddy with two inner vortices for circular, rhombus and square inner shapes and one vortex for triangular inner shape. For high Rayleigh numbers $\left(10^{5}\right.$ and $\left.10^{6}\right)$, the circulation of the flow becomes stronger. As a result, the maximum stream function values are increased from 5.835 to 18.390 for a circular shape, 7.947 to 19.421 for a square shape, 6.708 to 18.620 for a rhombus shape, and 7.996 to 20.654 for a triangular shape. The center of the lower vortex moves up for all inner shapes and approaches the top vortex until the two vortices merge. Heat transfer, on the other hand, is largely dominated by conduction at low Rayleigh, while convection heat transfer dominates within the physical domain and observes at high Rayleigh numbers the distorted isotherms and thinner boundary layers. The details are previously discussed.

Fig. 12 shows the effect of $\mathrm{Ra}$ on streamlines and isotherm contours with $\mathrm{N}=2$ at $\mathrm{AR}=5$ and $\varphi=0.05$ for different inner cylinder geometries. It is noted that for all the inner cylinder shapes, the Rayleigh number effect on streamlines and isotherms contours is very important than those for the previous state $(\mathrm{N}=1)$.
The streamlines and isotherms are parallel with each other particularly for those near the sinusoidal vertical walls of the enclosure and become more consistent with its shape. Also, for the case of $\mathrm{AR}=5$, the inner cylinder shapes are small as compared to the enclosure and the gap between the inner cylinder and enclosure is large. When $\mathrm{Ra}=10^{3}$, the primary eddy has a uni-cellular located in the bottom part of the enclosure for all inner shapes except for a triangular inner shape which has a bi-cellular. When $\mathrm{Ra}=10^{4}$, the primary eddy has a bi-cellular for circular, square, and rhombus cylinder shapes and a uni-cellular for triangular cylinder shape. The apparent change in streamline patterns can be due to the increased number of vertical wall undulations. The growth of the $\mathrm{Ra}\left(10^{5}\right.$ to $\left.10^{6}\right)$ causes a change in the flow patterns to increase. Owing to the increase in the strength of natural convection circulation within the space between the enclosure walls and inner cylinder, the primary eddy transitions from a bi-cellular to a uni-cellular at a high Rayleigh number. It can be observed from the results that when $\mathrm{Ra}$ increases from $10^{3}$ to $10^{6}$, values of the maximum stream function $\left(\Psi_{\max }\right)$ increase from $\left(\Psi_{\max }=0.13\right)$ to $\left(\Psi_{\max }=19.714\right)$ for a circular shape, $\left(\Psi_{\max }=0.13\right)$ to $\left(\Psi_{\max }=19.015\right)$ for square shape, $\left(\Psi_{\max }=0.133\right)$ to $\left(\Psi_{\max }=20.162\right)$ for rhombus shape and $\left(\Psi_{\max }=0.116\right)$ to $\left(\Psi_{\max }=21.402\right)$ for a triangular shape). The isotherm patterns match the behavior of streamlines. The isotherms are concentrated around all inner cylinder shapes at low $\mathrm{Ra}\left(10^{3}\right.$ and $\left.10^{4}\right)$. As Ra increases $\left(10^{5}\right)$, the isotherms are distorted and appear in a plume-like pattern over the cylinder. Further increase of $\mathrm{Ra}\left(10^{6}\right)$ the isotherm patterns depict the symmetrically for all inner cylinder shapes.

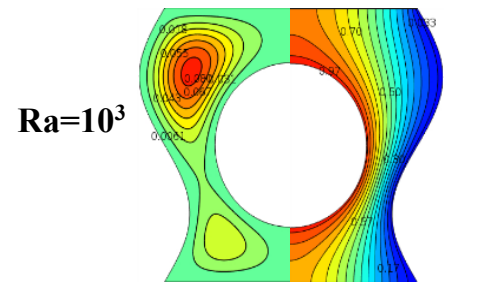

$\left|\Psi_{\text {max }}\right|=0.085$

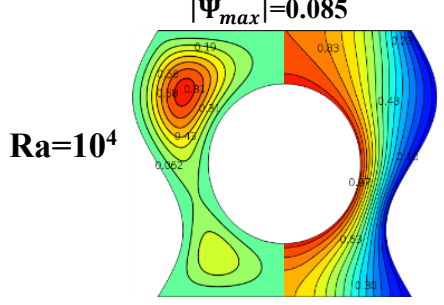

$\left|\Psi_{\max }\right|=\mathbf{0 . 8 6 7}$

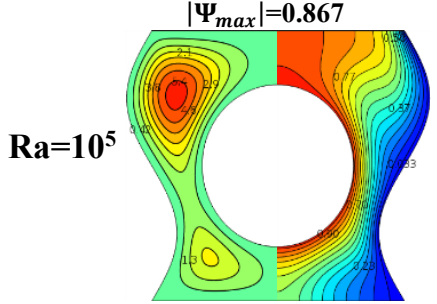

$\left|\Psi_{\max }\right|=\mathbf{5 . 8 3 5}$

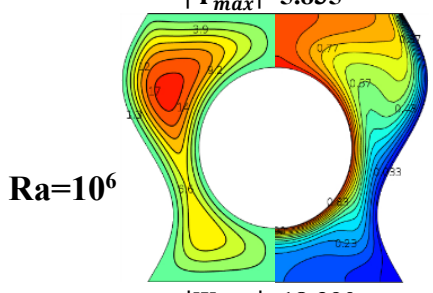

$\left|\Psi_{\max }\right|=18.390$

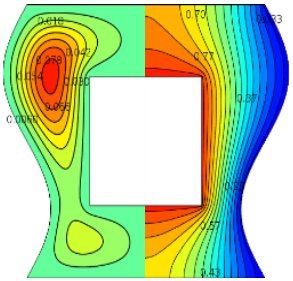

$\left|\Psi_{\max }\right|=\mathbf{0 . 1 3 8}$

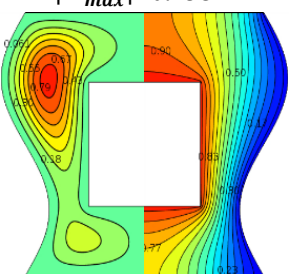

$\left|\Psi_{\text {max }}\right|=1.403$

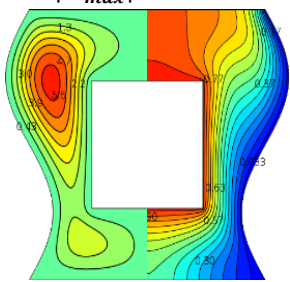

$\left|\Psi_{\max }\right|=\mathbf{7 . 9 4 7}$

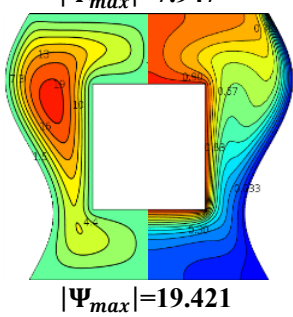

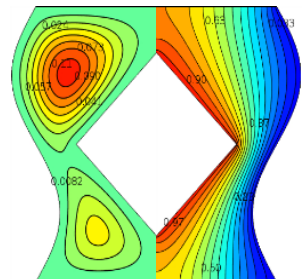

$\left|\Psi_{\max }\right|=0.114$

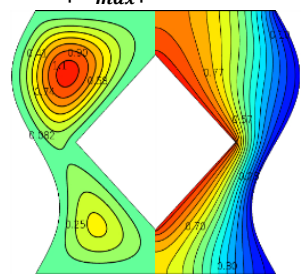

$\left|\Psi_{\max }\right|=1.151$

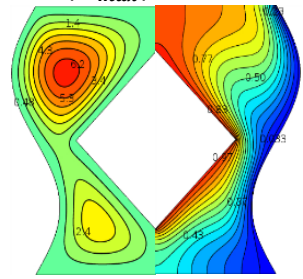

$\left|\Psi_{\max }\right|=6.708$

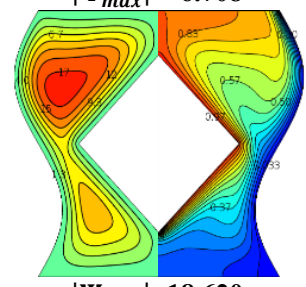

$\left|\Psi_{\max }\right|=18.620$

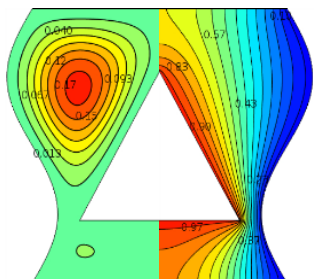

$\left|\Psi_{\text {max }}\right|=0.137$

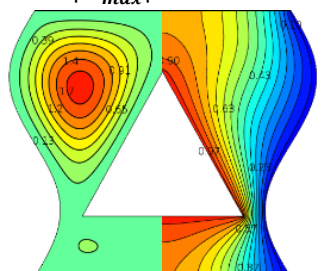

$\left|\Psi_{\max }\right|=\mathbf{1 . 3 8 6}$

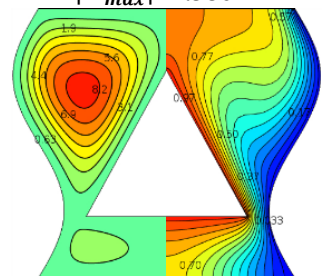

$\left|\Psi_{\max }\right|=7.996$

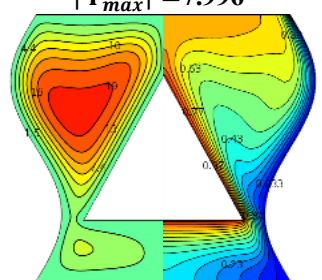

$\left|\Psi_{\max }\right|=\mathbf{2 0 . 6 5 4}$

Fig. 11 Streamlines (left) Isotherms (right) for a different Rayleigh number and geometry of inner cylinders at $\mathrm{AR}=1.67, \mathrm{~N}=1, \varphi=0.05$ 


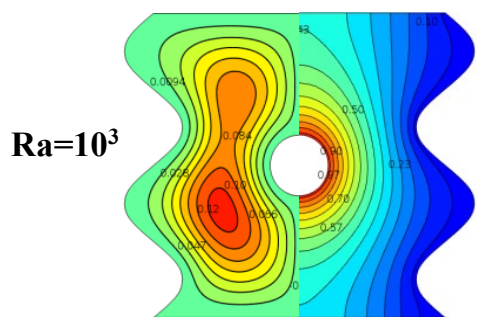

$\left|\Psi_{\max }\right|=\mathbf{0 . 1 3 0}$

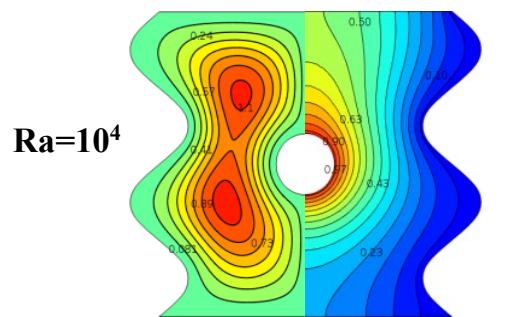

$\left|\Psi_{\max }\right|=1.133$

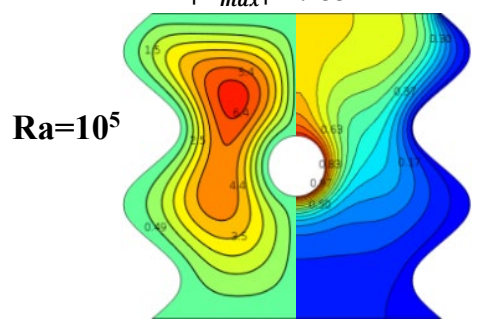

$\left|\Psi_{\max }\right|=6.914$

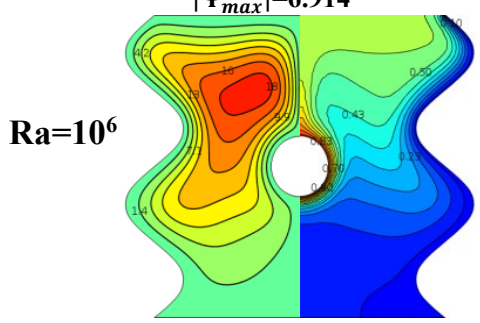

$\left|\Psi_{\max }\right|=19.714$

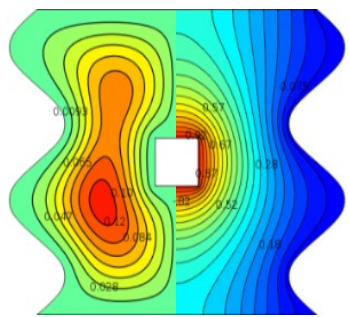

$\left|\Psi_{\text {max }}\right|=\mathbf{0 . 1 3 0}$

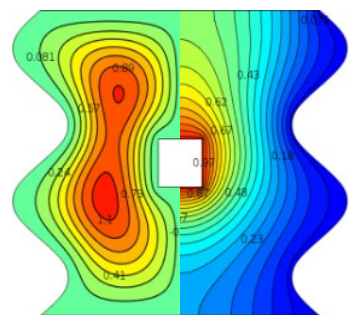

$\left|\Psi_{\text {max }}\right|=1.134$

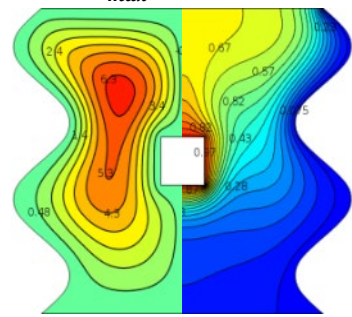

$\left|\Psi_{\max }\right|=6.740$

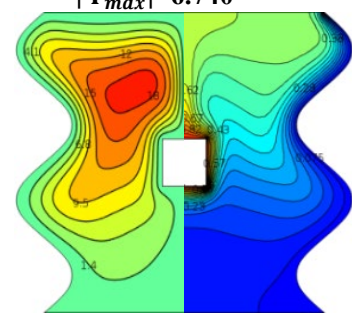

$\left|\Psi_{\max }\right|=19.015$

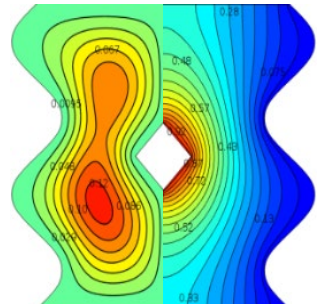

$\left|\Psi_{\text {max }}\right|=0.133$

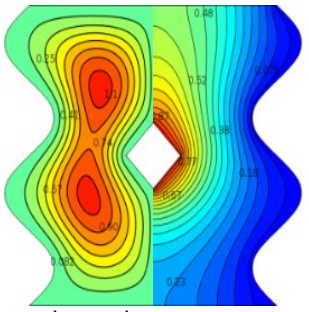

$\left|\Psi_{\text {max }}\right|=1.149$

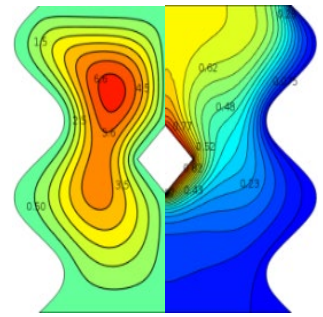

$\left|\Psi_{\max }\right|=\mathbf{7 . 0 6 8}$

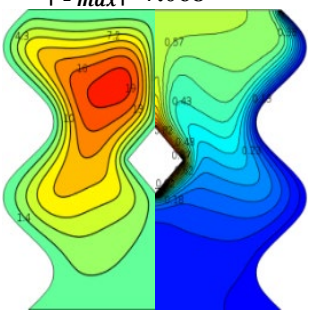

$\left|\Psi_{\max }\right|=20.162$

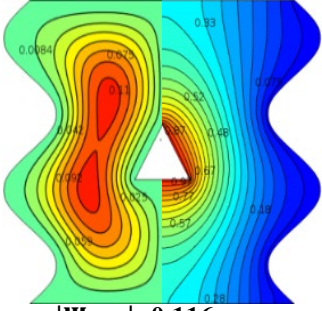

$\left|\Psi_{\max }\right|=\mathbf{0 . 1 1 6}$

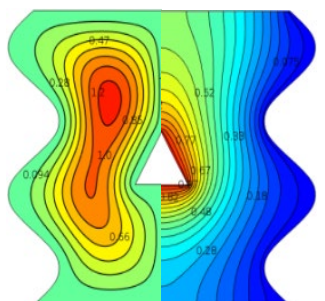

$\left|\Psi_{\max }\right| 1.313$

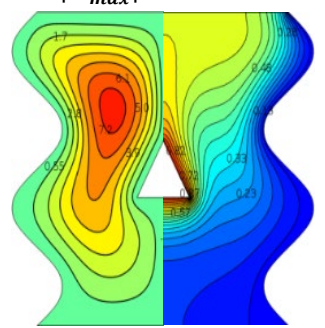

$\Psi_{\max } \mid=7.723$

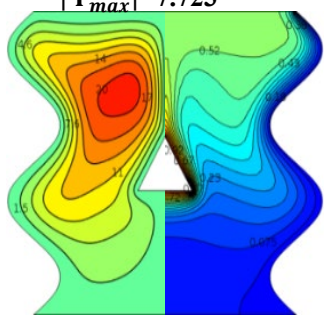

$\left|\Psi_{\max }\right|=21.402$

Fig. 12 Streamlines (left) Isotherms (right) for a different Rayleigh number and geometry of inner cylinders at AR=5, $\mathrm{N}=2, \varphi=0.05$

As shown in fig. 13, as the aspect ratio falls to 2.5 , the inner cylinder shapes become wider and the gap between the inner cylinder and the enclosure is decreased. In general, the patterns of streamline and isotherm for this case are the same as those for the aspect ratio of $\mathrm{AR}=5$. At the same time, the streamlines and isotherms are symmetrical in their patterns for all inner cylinder shapes as the Ra increases from $10^{3}$ to $10^{4}$, although the stream functions increase with $\mathrm{Ra}$ increases. The convection part plays a more important role in the heat transfer when the Ra rises to $10^{5}$ so that the isothermal patterns become unsymmetrical around the horizontal diagonal. Moreover, the values of the stream function increase because the buoyancy force becomes larger than that viscous force. At high $\mathrm{Ra}\left(10^{6}\right)$, the heat transfer is mainly dominated by convection. The core of the primary eddy moves upwards towards the cold surface of the enclosure. The behavior of the $\Psi_{\max }$ with the Ra for this case is the same as that for the case of $\mathrm{AR}=5$. That is, as the Ra increases, the $\Psi_{\max }$ increases in all inner cylinder shapes. But, if we compare the values of the maximum stream function for this case and the case of $\mathrm{AR}=5$ under the same Ra, we find that as the aspect ratio decreases, the $\Psi_{\max }$ is reduced. This shows that as the aspect ratio is reduced, the circulation of the flow becomes weaker, but the heat transfer in the enclosure domain becomes bigger.
The flow and thermal patterns in the enclosure domain are indicated in Fig. 14 when the aspect ratio decreases to 1.67. For all inner cylinder shapes considered, the streamline and isotherm patterns tend to be symmetrical at the low Rayleigh number $\left(10^{3}\right.$ and $\left.10^{4}\right)$. The flow inside the enclosure is relatively weak because of the greater viscous effects of the added surface area.

The streamlines exhibit two inner vortices within one primary eddy for all inner cylinder shapes except for a triangular cylinder shape. At high Rayleigh numbers $\left(10^{5}\right.$ and $\left.10^{6}\right)$, the strength of convective flow increases, and the isotherm patterns are distorted reflecting flow separation on top of the inner cylinder where the isotherms move rapidly away from the inner cylinder and form a thermal plume near the top. The stronger buoyancy-induced motion compensates for the added viscous effects due to the reduction in aspect ratio. The maximum stream function values are expected to increase with the increasing of Rayleigh numbers. But the streamline patterns remain relatively symmetrical while the isotherm patterns are unsymmetrical at these ranges of Rayleigh number. 

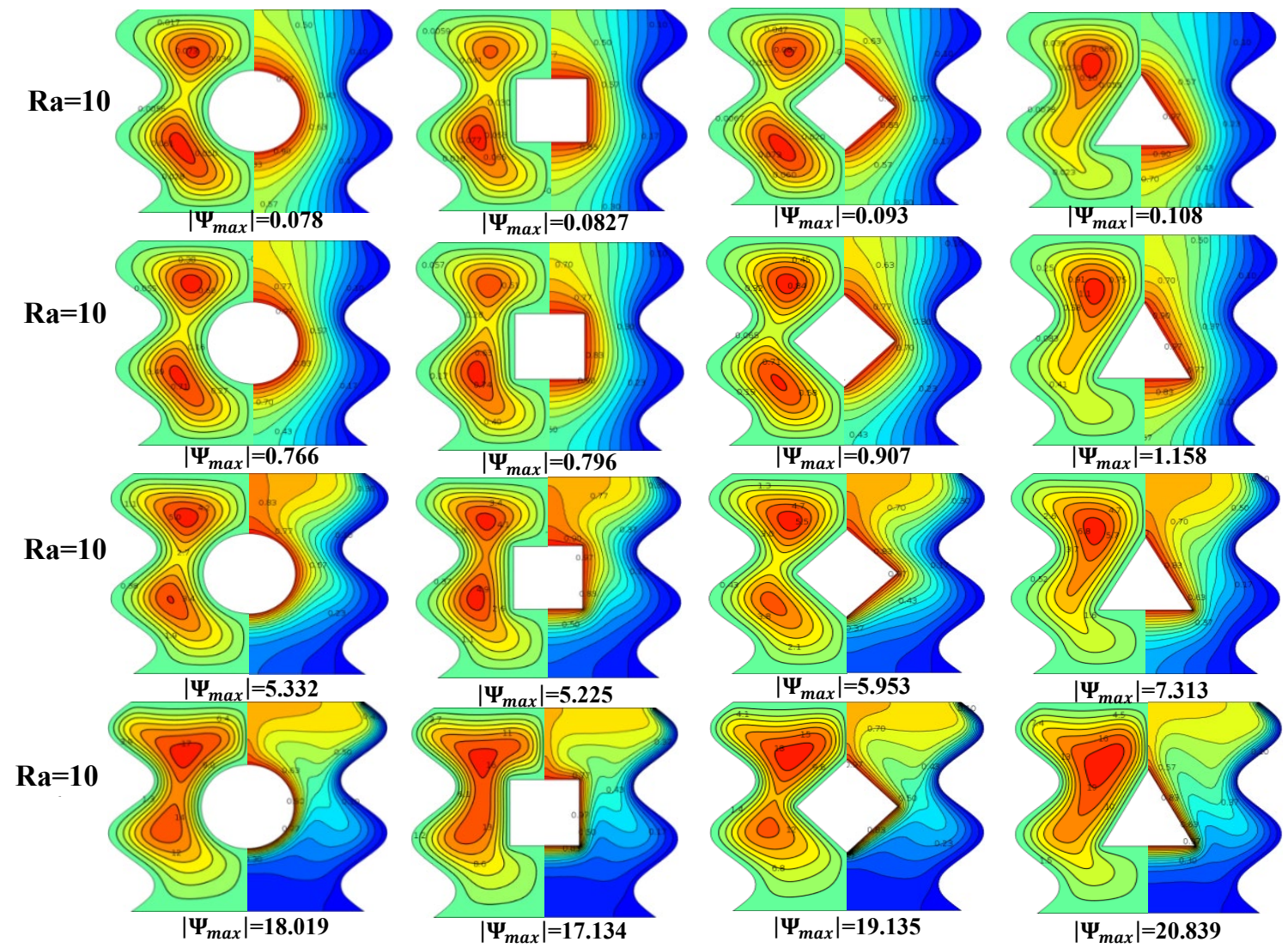

Fig. 13 Streamlines (left) Isotherms (right) for a different Rayleigh number and geometry of inner cylinders at $\mathrm{AR}=2.5, \mathrm{~N}=2, \varphi=0.05$
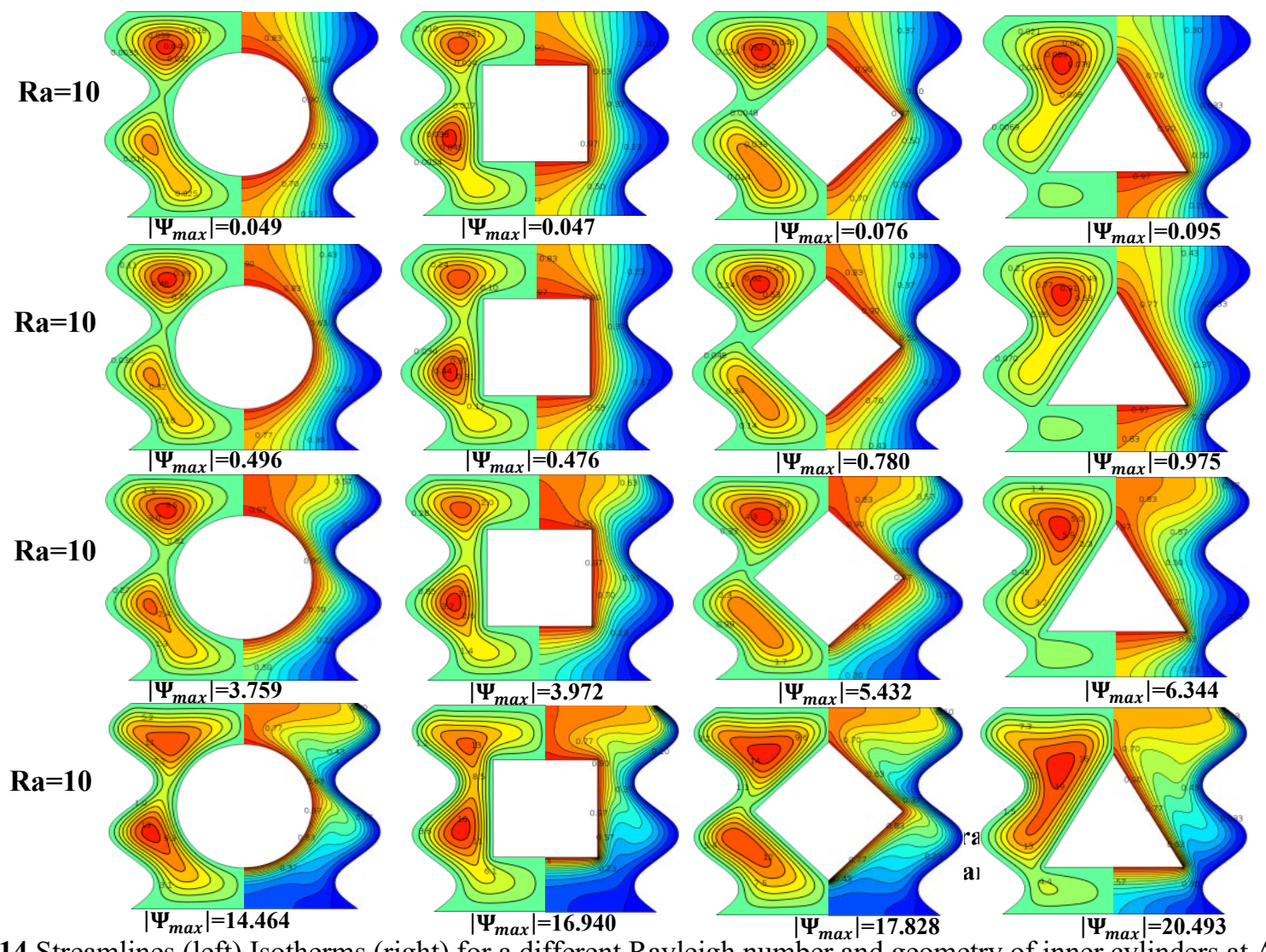

Fig. 14 Streamlines (left) Isotherms (right) for a different Rayleigh number and geometry of inner cylinders at $A R=1.67$, $\mathrm{N}=2, \varphi=0.05$ 
The variation of the local Nusselt number $\left(N u_{l}\right)$ around the hot inner cylinder is plotted as a function of the angular position (in radians) as presented in Fig. 15 for different shapes of cylinders, aspect ratios and undulation numbers at $\mathrm{Ra}=10^{6}$. When $\mathrm{Ra}=10^{6}, \mathrm{AR}=5 \varphi=0.05$, and $\mathrm{N}=0$, the figure displays a periodical fluctuation of the local Nusselt number at different inner cylinder shapes. Also, it can be seen that the sharp peak represented by the maximum value of $N u_{l}$ occurs specifically at $\theta_{\max }=$ $90^{\circ}$ for a circular shape, $\theta_{\max }=198^{\circ}$ for a square shape, $\theta_{\max }=205^{\circ}$ for a rhombus shape and $\theta_{\max }=240^{\circ}$ for a triangular shape. These peaks result from a high-temperature gradient at the heated surface of the inner cylinder. When AR decreases to 2.5 with the same previous conditions, the values of $N u_{l}$ are reduced for whole inner cylinder shapes.
Therefore, the peak for maximum heat transfer shifts back with the exception for a circular inner shape where $\theta_{\max } \cong 90^{\circ}$ for a circular shape, $\theta_{\max }=145^{\circ}$ for a square shape, $\theta_{\max }=150^{\circ}$ for a rhombus shape and $\theta_{\max }=$ $180^{\circ}$ for a triangular shape. With further decreasing in AR to 1.67, the reduction of $N u_{l}$ is continued and general shape of different curves is changed so that the peak of maximum heat transfer occurring at $\theta_{\max }=90^{\circ}$ for a circular shape, $\theta_{\max }=125^{\circ}$ for a square shape, $\theta_{\max }=130^{\circ}$ for a rhombus shape and $\theta_{\max }=315^{\circ}$ for a triangular shape. When the vertical walls of the enclosure are changed to a wavy at $\mathrm{N}=1$ with the same above conditions, formal distribution of $N u_{l}$ around the heated inner shapes for with exception of a triangular inner shape approximately remains constant. As the number of undulations increases from 1 to 2 , the undulation number on vertical walls does not affect the natural convection within the enclosure.
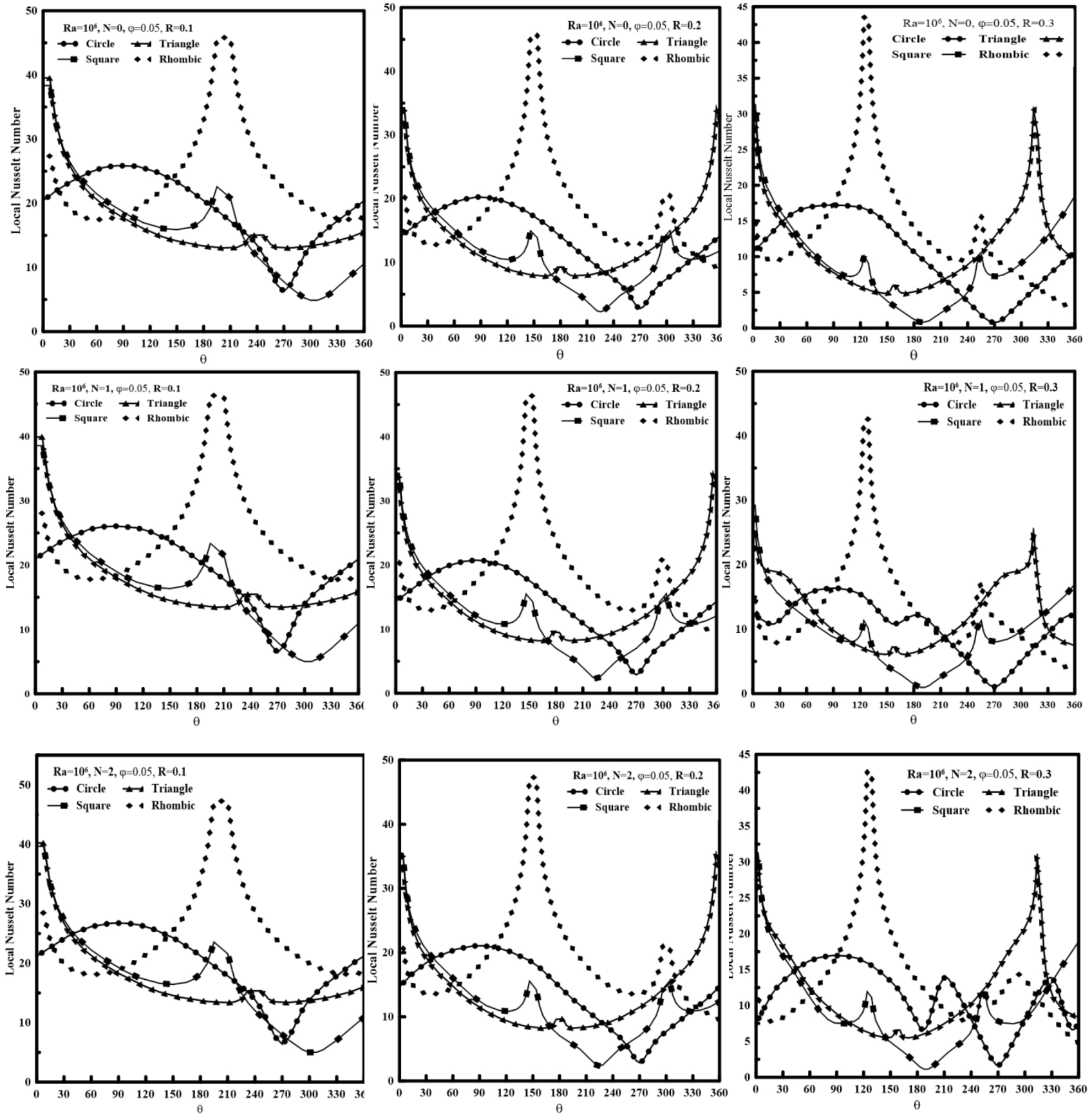

Fig. 15 Local Nusselt Number around hot inner cylinder for different shapes. 
Fig. 16 indicates the differences in the circular, cube, rhombus shape and triangular internal shapes of the average Nusselt number $(\bar{N} \bar{u})$ versus Rayleigh number. Due to the increased heat exchange between the fluid and the walls, it is noted that the average Nusselt number increases almost linearly with an increase of Ra for all the inner forms under the analysis. The results also indicate that the decrease in the aspect ratio of inner cylinder shapes leads to a decrease in the Nusselt number due to the temperature gradients diminish across the inner cylinder surface. Besides, the fluid flow within the smaller flow field becomes low so that the intensity of streamlines decreases. In the same figure, the $\overline{N u}$ is more elevated of the nanofluid $(\varphi=0.05)$ than that of the pure fluid $(\varphi=0)$ with increasing values of Rayleigh number. The explanation is that with the addition of nanoparticles in pure water, the heat capacity and thermal conductivity of silver-water nanofluid are enhanced. However, among the four kinds of inner cylinder forms, the heat transfer corresponding to the circular form reveals the maximum value of the heat transfer. In other words, the $\bar{N} \bar{u}$ of the circular inner cylinder is higher than that at rhombus, triangular and square shapes, respectively with different $\mathrm{Ra}$.
4. The heat conduction is predominant in the case of the small value of the Rayleigh number beside that the isotherms distributed evenly throughout the inner cylinder and the enclosure's corrugated walls. While in the vase of high value of $\mathrm{Ra}$, the fluid flow intensity goes up. Besides, deformation occurs in the patterns of isotherms and streamlines, signifying that in the space between the enclosure and the cylinder, natural convection has the largest contributor in the process of heat transfer.

5. For all inner cylinder types, the effect of adding the nanoparticles to the base fluid is improved by the heat transfer rates.

6. The triangular inner cylinder shape inside the corrugated enclosure has slightly higher stream functions than the others.

7. It is concluded that Nusselt number enhances as both of the aspect ratio as well as Rayleigh number increases for all of the selected diverse inner shapes.
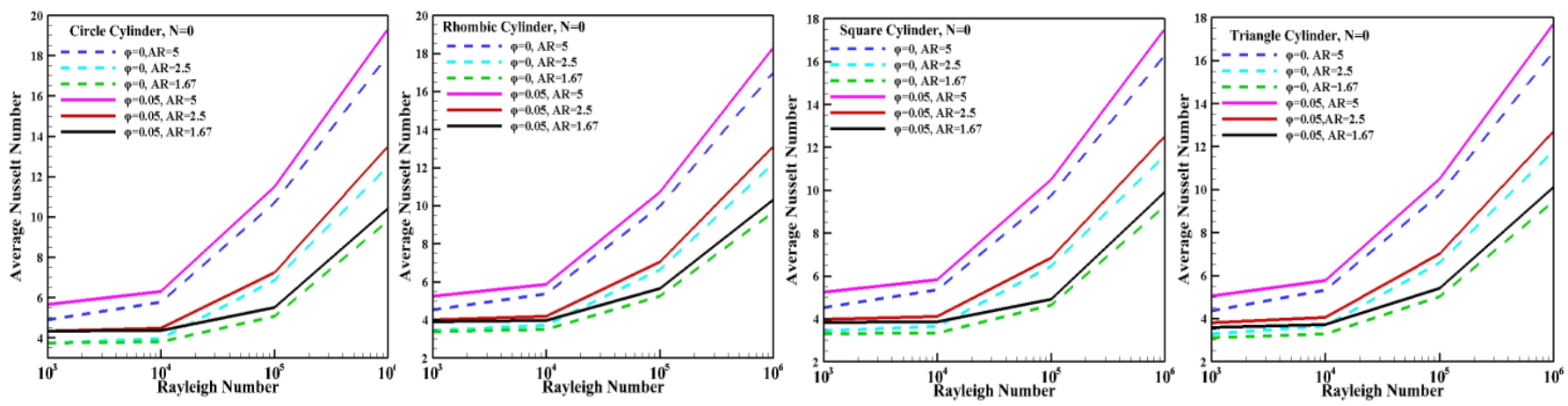

Fig. 16 Average Nusselt Number of hot inner cylinders for different shapes.

\section{CONCLUSION}

In this research, characteristics of internal thermal driven flow of heat transfer within the corrugated enclosure of sinusoidal wave including concentric inner cylinder are analyzed for a steady-state, laminar and incompressible flow. This enclosure is filled with silver/water nanofluid as a working fluid. Adiabatic conditions had been applied to the horizontal walls. However, the vertical walls are keeping at isotherm cold temperature, while the isotherm hot conditions of temperature are applied to the internal circular cylinder. Effects of various geometric shapes of the inner cylinder (circular, square, rhombus and triangular) on the flow and thermal fields inside the enclosure are carried out for Rayleigh numbers and undulation numbers of vertical walls. The obtained results from this study are:

1. There is a clear impact on the flow fields and temperature of the aspect ratio and Rayleigh number. As $\mathrm{Ra}$ and aspect ratio increase, the convection heat transfer is increased for circular, rhombus, square, and triangular forms, respectively.

2. The effect of the increase of the undulation numbers on the HT rate is considerable for all inner cylinder shapes at higher $\mathrm{Ra}\left(\mathrm{Ra}=10^{6}\right)$. While the decrease of the aspect ratios has reduced the heat transfer rate for all inner cylinder shapes.

3. The circular inner cylinder has the maximum heat transfer among the four types of inner cylinder shapes.

\section{NOMENCLATURE}

$\mathrm{Cp} \quad$ Specified heat measured at the steady pressure $(\mathrm{kJ} / \mathrm{kg} . \mathrm{K})$

g Acceleration of Gravitaty $(\mathrm{m} / \mathrm{s} 2)$

k Thermal conductivity (W/m.K)

$\mathrm{P} \quad$ Pressure without Dimension

$\mathrm{p} \quad$ Pressure $(\mathrm{Pa})$

Pr Prandtl number ( $v f / \alpha f)$

$\mathrm{R} \quad$ Radius of circular cylinder (m)

$\mathrm{Ra} \quad$ Rayleigh number

T Temperature (K)

Tc The cold surface's Temperature (K)

M Total number of nodes

L Length and height of Enclosure

YO Distance between original and wavy wall

Th The temperature of the hot surface's (K)

$\mathrm{u} \quad$ Velocity component in $\mathrm{x}$-axis $(\mathrm{m} / \mathrm{s})$

$\mathrm{y} \quad$ vertical-axis $(\mathrm{m})$ of the cartesian coordinate

$\mathrm{V} \quad$ The component of Dimensionless velocity in y-axis

$\mathrm{v} \quad$ Velocity component in y-axis $(\mathrm{m} / \mathrm{s})$

$\mathrm{X} \quad$ Dimensionless horizontal- axis

$\mathrm{X}$

$\mathrm{Y}$

$\mathrm{U}$

$\mathrm{Nul}$

$\mathrm{N}$
Horizontal direction in the cartesian coordinates (m)

Dimensionless axis in the vertical direction

Dimensionless Velocity in $\mathrm{x}$-axis $(\mathrm{m} / \mathrm{s})$

Localized number of Nusselt the hot

Undulation number 
$\begin{array}{ll}\text { AR } & \text { Aspect ratio } \\ \text { Nuave } & \text { Average Nusselt number }\end{array}$

Greek symbols

$\alpha \quad$ The diffusivity of heat ( $\mathrm{m} 2 / \mathrm{s})$

$\psi \quad$ Dimensionless stream function

$\mathrm{T}^{*} \quad$ The dimensionless temperature (T-Tc/Th- $\left.\mathrm{Tc}\right)$

$\Psi \quad$ The dimensional stream function $(\mathrm{m} 2 / \mathrm{s})$

$\mu \quad$ Dynamic viscosity $(\mathrm{kg} / \mathrm{ms})$

$\beta \quad$ Thermal expansion Volumetric coefficient (K-1)

$\rho \quad$ Density $(\mathrm{kg} / \mathrm{m} 3)$

$\varphi \quad$ Nanofluid volume fraction

$\nu \quad$ Kinematic viscosity $(\mu / \rho)(\mathrm{m} 2 \mathrm{~s}-1)$

$\lambda \quad$ Amplitude

$\gamma \quad$ Penalty Parameter

$\theta \quad$ Angle of rotation

$\Gamma \quad$ Basis function

Subscripts

fl Pure Fluid

c Cold

h Hot

sp solid

nfl Nanopartical

I Node number

i Residual number

\section{References}

Ahmed N., Vieru D., Fetecau C., and Shah A., 2018, "Convective flows of generalized time-nonlocal nanofluids through a vertical rectangular channel”, Phys. Fluids Vol.30, 052002.

https://doi.org/10.1063/1.5032165

Alsabery, A.I., Tayebi T., Chamha ALI J., and Hashim I., 2018, "Effect of rotating solid cylinder on entropy generation and convective heat transfer in a wavy porous cavity heated from below", International Communications in Heat and Mass Transfer, Vol. 95, pp. 197-209. https://doi.org/10.1016/j.icheatmasstransfer.2018.05.003

Al-Amir Qusay Rasheed, Alinnawi Farooq Hassan Ali, and Hamza Hameed Kadeem, 2017, "Effect of wavy wall location on the natural convection in an enclosure containing a concentric heated circular cylinder", The Iraqi Journal For Mechanical And Material Engineering, Vol.17, No. 2, pp.291-308.

https://doi.org/10.1615/2011003270

Aminossadati, S. and Ghasemi B., 2009,'Natural convection cooling of a localised heat source at the bottom of a nanofluid-filled enclosure", European Journal of Mechanics-B/Fluids, Vol. 28, No. 5, pp. 630-640. https://doi.org/10.1016/j.euromechflu.2009.05.006

Baytas A.C. and Pop I.,1999," Free convection in oblique enclosures filled with a porous medium", International Journal of Heat and Mass Transfer, Vol.42, No.6, pp. 1047-1057.

https://doi.org/10.1016/s0017-9310(98)00208-7

Bin Kim, J.M.H., Ho Sang Kwak, GI, 2001,'Buoyant convection in a square cavity partially filled with a heat-generating porous medium", Numerical Heat Transfer: Part A: Applications, Vol.40, No. 6, p. 601618. https://doi.org/10.1080/10407780152655388
Celli M., 2013,"Non-homogeneous model for a side heated square cavity filled with a nanofluid", International Journal of Heat and Fluid Flow, Vol.44, pp. 327-335.

https://doi.org/10.1016/j.ijheatfluidflow.2013.07.002

Chamkha Ali J., Hussain Salam Hadi, 2011,"AL-AMIR QUSAY RASHEED, Mixed Convection Heat Transfer of Air inside a Square Vented Cavity with a Heated Horizontal Square Cylinder", Numerical Heat Transfer, Part A: Applications, No. 59, pp. 58-79. https://doi.org/10.1080/10407782.2011.541216

Choi C., Jeong S., Yeong HA. M., Sik Yoon H.,2014," Effect of a circular cylinder's location on natural convection in a rhombus enclosure", International Journal of Heat and Mass Transfer, Vol. 77, pp. 60-73. https://doi.org/10.1016/j.ijheatmasstransfer.2014.04.071

Cho, C.-C. and Chen C.-L., 2012," Natural convection heat transfer performance in complex-wavy-wall enclosed cavity filled with nanofluid", International Journal of Thermal Sciences, Vol. 60, pp. 255263. https://doi.org/10.1016/j.ijthermalsci.2012.05.001

Cho, C.-C., Chiu C.-H., and Lai C.-Y., 2016," Natural convection and entropy generation of A12O3-water nanofluid in an inclined wavy-wall cavity", International Journal of Heat and Mass Transfer, Vol. 97, pp. 511-520 . https://doi.org/10.1016/j.ijheatmasstransfer.2016.01.078

Colanglo G., Favale E., Millanese M., De Risi A., and Lafororgia D., 2017,'Cooling of electronic devices: Nanofluids contribution", Applied Thermal Engineering, Vol. 127, pp. 421-435.

https://doi.org/10.1016/j.applthermaleng.2017.08.042

Esmaeilpour M. and Abdollahadeh M., 2012, "Free convection and entropy generation of nanofluid inside an enclosure with different patterns of vertical wavy walls", International Journal of Thermal Sciences, Vol. 52, pp. 127-136.

https://doi.org/10.1016/j.ijthermalsci.2011.08.019

Ghasemi B. and Aminossadati S.M., 2010," Periodic natural convection in a nanofluid-filled enclosure with oscillating heat flux", International Journal of Thermal Sciences, Vol. 49, pp. 1-9 (2010).

https://doi.org/10.1016/j.ijthermalsci.2009.07.020

Ghasemi, B. and Aminossadati S.M., 2010," Brownian motion of nanoparticles in a triangular enclosure with natural convection”, Int. J. Thermal Sci., Vol. 49, pp. 931-940.

https://doi.org/10.1016/j.ijthermalsci.2009.12.017

H. Astuti, P. Sri, and S. Kaprawi, 2019, "Natural convection of nanofluid past an accelerated vertical plate with variable wall temperature by presence of the radiation", Frontiers in Heat and Mass Transfer, Vol.13, No.3, pp. 1-8.

https://doi.org/10.5098/hmt.13.3.

House John M., Beckerman Christoph and Smith Theodor F., 1990," Effect of a centered conducting body on natural convection heat transfer in an enclosure", Numerical Heat Transfer, Part A, vol. 18, pp. 213-225. https://doi.org/10.1080/10407789008944791

Humaira Tasnim S., Mahmud S., and Das P.K., 2002, "Effect of aspect ratio and eccentricity on heat transfer from a cylinder in a cavity", 
International Journal of Numerical Methods for Heat and Fluid Flow, Vol. 12, No. 7, pp. 855-869.

https://doi.org/10.1108/09615530210443061

Hussin M. Jassim, Ali. Farooq H., Al-Amir Qusay Rasheed, Hamzah Hameed K., Khafaji Salwan Obaid Waheed, 2019, "Entropy generation analysis of a natural convection inside a sinusoidal enclosure with different shapes of cylinders", Frontiers in Heat and Mass Transfer, Vol.12, No.22, pp. 1-9. https://doi.org/10.5098/hmt.12.22

Hussain Salam Hadi and Al-Amir Qusay Rasheed, 2011" Mixed convection heat transfer flow of air inside a sinusoidal corrugated cavity with a heat-conducting horizontal circular cylinder, Journal of Enhanced Heat Transfer, Vol. 18, No. 5, pp. 433-447.

https://doi.org/10.1615/jenhheattransf.2011003270

Hussain S. H. and A.K. Hussen, 2010," Numerical investigation of natural convection phenomena in a uniformly heated circular cylinder immersed in square enclosure filled with air at different vertical locations". International Communications in Heat and Mass Transfer, Vol. 37,No. 8, pp. 1115-1126.

https://doi.org/10.1016/j.icheatmasstransfer.2010.05.016

J. N. Reddy, 1993,"An Introduction to the finite element method", McGraw-Hill, New York.

https://doi.org/10.1299/jsmemag.82.732_1234

Jou R. Y. and Tzeng S. C., 2006," Numerical research of nature convective heat transfer enhancement filled with nanofluids in rectangular enclosures", International Communications in Heat and Mass Transfer, Vol. 33, No. 6, pp. 727-736.

https://doi.org/10.1016/j.icheatmasstransfer.2006.02.016

Joudi K. A., Hussein I. A., Farhan A. A., 2004," Computational model for a prism shaped storage solar collector with a right triangular cross section, Energy Conversion and Management. 45, pp.337-342. https://doi.org/10.1016/s0196-8904(03)00153-5

Kang, D.H., HA M. Y., Yoon H. S., 2013," Chang young Choi Bifurcation to unsteady natural convection in square enclosure with a circular cylinder at Rayleigh number of 107', International Journal of Heat and Mass Transfer, Vol. 64, pp. 926-944.

https://doi.org/10.1016/j.ijheatmasstransfer.2013.05.002

Khanafer K., Vafai K., and Lightone M., 2003,” Buoyancy-driven heat transfer enhancement in a two-dimensional enclosure utilizing nanofluids. International journal of heat and mass transfer", Vol. 46, No. 19, pp. 3639-3653. https://doi.org/10.1016/s0017-9310(03)00156-x

Kherbeet ASh., Mohammed HA, Salman, Ahmed Hamdi E, and Allawi Omer A., 2014," Experimental and numerical study of nanofluid flow and heat transfer over microscale backward-facing step". International Journal of Heat and Mass Transfer, Vol. 79, pp. 858-67. https://doi.org/10.1016/j.ijheatmasstransfer.2014.08.074

Khoshvaght Aliabadi M., 2014," Influence of different design parameters and A12O3-water nanofluid flow on heat transfer and flow characteristics of sinusoidal-corrugated channels", Energy conversion and management, Vol. 88, pp. 96-105.

https://doi.org/10.1016/j.enconman.2014.08.042
Khohvaght-Aliabadi M., Hassani S., and Maloumi S.H., 2017," Comparison of hydrothermal performance between plate fins and platepin fins subject to nanofluid-cooled corrugated miniature heat sinks". Microelectronics Reliability, Vol. 70, pp. 84-96.

https://doi.org/10.1016/j.microrel.2017.01.005

Khohvaght-Aliabadi, M., A. Zamzamian, and F. Hormoi, 2014," Wavy channel and different nanofluids effects on performance of plate-fin heat exchangers", Journal of Thermophysics and Heat Transfer, Vol. 28, No. 3, pp. 474-484. https://doi.org/10.2514/1.t4209

Kumar, S., Prasad S.K., and Banerjee, 2010," Analysis of flow and thermal field in nanofluid using a single phase thermal dispersion model", Applied Mathematical Modelling, Vol. 34, No. 3, pp. 573-592. https://doi.org/10.1016/j.apm.2009.06.026

Lee J., Ha M., and Yoom H., 2010, " Natural convection in a square enclosure with a circular cylinder at different horizontal and diagonal locations", International Journal of Heat and Mass Transfer, Vol. 53, No. 26, pp. 5905-5919.

https://doi.org/10.1016/j.ijheatmasstransfer.2010.07.043

Majdi H.S., Abdulkadim A., and Abed A.M., 2019," Numerical investigation of natural convection heat transfer in a parallelogramic enclosure having an inner circular cylinder using cylinder using liquid nanofluid", Frontier Heat and Mass Transfer, 12, 1-14.

https://doi.org/10.5098/hmt.12.2

Mohammed K.A., Talib A.R. Abu, Nuraini A.A., Ahmed K.A., 2017," Review of forced convection nanofluids through corrugated facing step". Renewable and Sustainable Energy Reviews, Vol. 75, pp. 234-241. https://doi.org/10.1016/j.rser.2016.10.067

Mohmud H. Ali, Rawand E. Jalal, 2020, "Natural convection in a square enclosure with different openning and involves two cylinders: a numerical approach", Frontiers in Heat and Mass Transfer, Vol.15, No.27, pp. 1-14, https://doi.org/10.5098/hmt.15.27

Murshed S.S. and De Castrro C.N., 2016," Conduction and convection heat transfer characteristics of ethylene glycol based nanofluids-a review", Applied energy, Vol. 184, pp. 681-695.

https://doi.org/10.1016/j.apenergy.2016.11.017

Ostrach S., 1988,"Natural convection in enclosures", J. Heat Transfer 110, pp.1175-1190. https://doi.org/10.1016/S0065-2717(08)70039-X

Rafiq Manna, Patric H. Oosthuien, 2019, "Numerical and experimental investigations of natural convection heat transfer from two-sided diagonally inclined square plates having a finite thickness", Frontiers in Heat and Mass Transfer, Vol.13, No.7, pp. 1-12, https://doi.org/10.5098/hmt.13.7

Ridha Jmai, Ben-Beya Brahim, and Lili Taieb, 2013," Heat transfer and fluid flow of nanofluid-filled enclosure with two partially heated side walls and different nanoparticles", Super lattices and Microstructures, Vol. 53, pp. 130-154.

https://doi.org/10.1016/j.spmi.2012.10.003 
Roslan., Saleh H., and Hashim I., 2014," Natural convection in a differentially heated square enclosure with a solid polygon", The Scientific World Journal.

http://dx.doi.org/10.1155/2014/617492

Salam Hadi Hussain \& Qusay Rashid Abd-Amer, 2011, " Mixed Convection Heat Transfer Flow Of Air Inside A Sinusoidal Corrugated Cavity With A Heat-conducting Horizontal Circular Cylinder", Journal of Enhanced Heat Transfer, 18 (5): 433-447.

https://doi.org/10.1615/JEnhHeatTransf.2011003270

Sheremet M.A., S. Dinarvand, and I. Pop, 2015 "Effect of thermal stratification on free convection in a square porous cavity filled with a nanofluid using Tiwari and Das' nanofluid model. Physica E: LowDimensional Systems and Nanostructures, No. 69, pp. 332-341. https://doi.org/10.1016/j.physe.2015.02.005

Shenoy A., Sheremet M., and Pop I., 2016," Convective flow and heat transfer from wavy surfaces: viscous fluids, porous media, and nanofluids", CRC Press . https://doi.org/10.1201/9781315367637

Sheikholeslami M., Shehad S.A., Li Z., and Shafee A., 2018," Numerical modeling for alumina nanofluid magnetohydrodynamic convective heat transfer in a permeable medium using Darcy law". International Journal of Heat and Mass Transfer, Vol. 127, pp. 614-622. https://doi.org/10.1016/j.ijheatmasstransfer.2018.07.013

Sheikholeslami M., and Oztop HF., 2017," MHD free convection of nanofluid in a cavity with sinusoidal walls by using CVFEM", Chin J Phys., Vol. 55, No. 6, pp2291-2304.

https://doi.org/10.1016/j.cjph.2017.09.006

Shirvan K. M., Ellah R., Mamourian M., Moghiman M., 2017, "Effects of wavy surface characteristics on natural convection heat transfer in a cosine corrugated square cavity filled with nanofluid", International Journal of Heat and Mass Transfer, Vol. 107, pp. 1110-1118. https://doi.org/10.1016/j.ijheatmasstransfer.2016.11.022

Shu C., Xye H. and Zhu Y., 2001, "Numerical study of natural convection in an eccentric annulus between a square outer cylinder and a circular inner cylinder using DQ method", International Journal of Heat and Mass Transfer, Vol. 44, No. 17, pp. 3321-3333.

https://doi.org/10.1016/s0017-9310(00)00357-4

Tanmay Bask, and Chamkha Ali J., 2012," Heatline analysis on natural convection for nanofluids confined within square cavities with various thermal boundary conditions", International Journal of Heat and Mass Transfer, 55, 5526-5543.

https://doi.org/10.1016/j.ijheatmasstransfer.2012.05.025

Tanmay Basak,, S. Roy, and A. R. Balakrishnamn, 2006," Effects of thermal boundary conditions on natural convection flows within a square cavity", Int. J. Heat and Mass Transfer, 49, 4525-4535. https://doi.org/10.1016/j.ijheatmasstransfer.2006.05.015

Tiwari R.K. and Das M.K., 2007," Heat transfer augmentation in a twosided lid-driven differentially heated cavity utilizing nanofluids", International Journal of Heat and Mass Transfer, Vol. 50, No. 10, pp. 2002-2018.

https://doi.org/10.1016/j.ijheatmasstransfer.2006.09.034

Xu X, Sun G, Yu Z, Hu Y, Fanl, Cen K., 2009,’Numerical investigation of laminar natural convective heat transfer from a horizontal triangular cylinder to its concentric cylindrical enclosure". Int J Heat Mass Transfer. Vol.52, No. (13-14), pp.3176-3186. https://doi.org/10.1016/j.ijheatmasstransfer.2009.01.026 